Pacific Journal of Mathematics

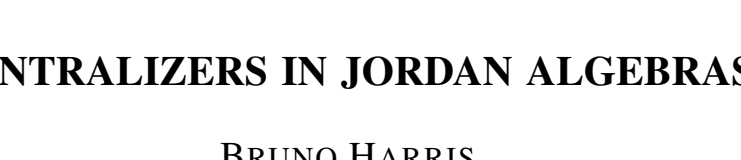




\title{
CENTRALIZERS IN JORDAN ALGEBRAS
}

\author{
BRUNO HARRIS
}

Introduction. The aim of this paper is to prove for Jordan algebras some theorems on centralizers of subalgebras analogous to known results in the theory of associative algebras (contained in [6, Chapter 3] and [7, Chapter 6], for instance).

The definition of the centralizer of a subalgebra in a Jordan algebra is based on the concept of "operator commutativity" introduced by Jordan, von Neumann and Wigner in [17]: two elements $x, y$ of the Jordan algebra $J$ operator commute if the operators $R_{x}: a \rightarrow a x$ and $R_{y}: a \rightarrow a y$, acting on $J$, commute, that is $(a x) y=(a y) x$ for all elements $a$ of $J$. In $\S 1$ we study this concept, extend the results of [8] to algebras over fields of characteristic not two, and show that for many types of Jordan algebras obtained from associative algebras by introducing the Jordan product $a \circ b=a b+b a$ ( $a b$ the associative product), the centralizer of a subalgebra is just the set of elements commuting in the associative multiplication with the elements of the subalgebra. Thus some of our later results can be regarded as generalizations of the associative algebra results if we convert the associative algebras into Jordan algebras by means of the Jordan product.

In $\S 2$ we generalize some of the theory of a single linear transformation in a finite dimensional vector space (see [6, Chapter 3] and [13]) to the subalgebra generated by a single element in a simple finite dimensional Jordan algebra. We show that such a subalgebra is equal to the centralizer of its centralizer, and we also generalize to any central simple Jordan algebra a formula of Frobenius giving the dimensionality of the centralizer of a single linear transformation in terms of the degrees of its invariant factors. A special case of this formula-namely, the formula for the central simple Jordan algebra of all symmetric matrices-was proved earlier, and by a different method, by H. Osborn (to appear in these Transactions).

In $\S 3$ we study the centralizer theory of a simple subalgebra in a central simple Jordan algebra. We show that the analogues of the centralizer and double centralizer theorems for simple finite dimensional subalgebras of the associative algebra of all linear transformations on a vector space ([15]) also hold for simple finite dimensional Jordan sub-

This paper was originally accepted by the Transactions of the American Mathematical Society, Received July 10, 1957, by Trans. Amer. Math. Soc. Most of the material of this paper is contained in the author's doctoral dissertation, Yale University, 1956. Some of the results were presented to the Society in October, 1955 and April, 1957. The author is a National Science Foundation Postdoctoral Fellow, 1956-57. 
algebras of the Jordan algebra of all self-adjoint linear transformations on a vector space with inner product. Incidentally we show that some of the results of [15] can be generalized from the class of rings of all continuous linear transformations on a vector space to the larger class of primitive rings with minimal ideals. In the same way the Galois theory of automorphisms of [16] can be generalized to primitive rings with minimal ideals.

In conclusion we would like to express our gratitude to Professor Nathan Jacobson who suggested these problems and gave much stimulating advice.

Preliminary Notions. A Jordan algebra is a linear algebra, whose multiplication we shall denote by $x \circ y$, satisfying the following identities

$$
\begin{aligned}
& x \circ y=y \circ x \\
& ((x \circ x) \circ y) \circ x=(x \circ x) \circ(y \circ x) .
\end{aligned}
$$

We shall always assume that the base field has characteristic different from 2 .

A special Jordan algebra is a subspace of an associative algebra (with associative multiplication $x y$ ) closed under the composition $x \circ y=$ $x y+y x$. The special Jordan algebra whose underlying vector space coincides with that of the associative algebra $\mathfrak{A}$ and whose multiplication is $x \circ y=x y+y x$ ( $x y$ the multiplication in $\mathfrak{A}$ ) will be denoted by $\mathfrak{A}_{j}$. If $\mathfrak{A}$ is an associative algebra with an involution, the subset of elements left fixed by the involution is also a special Jordan algebra, which will be denoted by $H(\mathfrak{U})$. The same notation will be used for the set of elements left fixed by an involution in a possibly non-associative algebra $\mathfrak{A}$ : this set may or may not be a Jordan algebra.

We shall have to consider sometimes matrix algebras with coefficients in a (possibly non-associative) algebra with identity element. A set of matrix units in an algebra of all $n \times n$ matrices $(n \geqq 2)$ will mean a set of elements $e_{i j}, i, j=1, \cdots, n$ which associate with every pair of elements of the algebra (i.e. lie in the nucleus) and satisfy

$$
\begin{aligned}
& e_{i j} e_{k l}=\delta_{j k} e_{i l}\left(\delta_{j k}\right. \text { the Kronecker delta) } \\
& e_{11}+\cdots+e_{n n}=1 \text {, the identity element. }
\end{aligned}
$$

If we consider Jordan algebras (with identity) of all hermitian matrices with coefficients in an involutorial algebra we are led to consider elements (which we shall also call matrix units) $e_{i i}, u_{i j}$ with $i<j, i, j=1, \ldots, n$, $n \geqq 3$, such that

$$
\begin{aligned}
& e_{i i} \circ e_{i i}=2 e_{i i}, \quad e_{i i} \circ u_{i j}=u_{i j}, \quad u_{i j} \circ u_{i j}=2\left(e_{i i}+e_{j j}\right) \\
& u_{i j} \circ u_{j k}=u_{i k} \text { if } i, j, k \text { are distinct, } \sum e_{i i}=1
\end{aligned}
$$


and all other products are zero. As shown in [9], Th. 9.1, any set of elements $e_{i i}, u_{i j}$ satisfying (4) leads to a representation of the Jordan algebra as the subalgebra of $n \times n$ hermitian matrices of an algebra $\mathfrak{A}_{\text {, }}$ where $\mathfrak{A}$ is the algebra of all $n \times n$ matrices with coefficients in an involutorial algebra, and, if $f_{i j} ; i, j=1, \ldots, n$ are the matrix units in $\mathfrak{A}$, then

$$
f_{i i}=e_{i i}, \quad u_{i j}=f_{i j}+f_{j i}
$$

If the base field is algebraically closed and $\mathfrak{\Im}$ is the exceptional simple Jordan algebra of all $3 \times 3$ hermitian matrices with coefficients in the Cayley algebra, then given elements $e_{i i}, i=1,2,3$, in $\mathfrak{\Im}$, satisfying $e_{i i} \circ e_{j j}=2 \delta_{i j} e_{i j}$ we can find elements $u_{12}, u_{13}$ (in many different ways) satisfying $u_{12} \circ u_{12}=2\left(e_{11}+e_{22}\right), u_{13} \circ u_{13}=2\left(e_{11}+e_{33}\right)$ such that the $e_{i i}, u_{12}, u_{13}$ and $u_{23}=u_{12} \circ u_{13}$ satisfy the conditions (4) and hence are the "matrix units" of another representation of $\mathfrak{\Im}$ as $3 \times 3$ hermitian matrices with Cayley number coefficients.

Finally we shall summarize briefly the classification of the finite dimensional central simple Jordan algebras. For further references about classification, structure, or representation theory of Jordan algebras, one should consult [9].

First, assume the base field algebraically closed. Then the algebra has an identity element; if the identity element can be written as a sum of $n$, but not more, mutually orthogonal idempotents, then the algebra is said to have degree $n$. ( $e$ is an idempotent if $e \circ e=e$ ).

If $n=1$, then the algebra is one-dimensional, [10]. If $n=2$, the algebra is a vector space direct sum of the subspace generated by 1 and of a vector space $V$ of dimension at least 2 with non-degenerate symmetric scalar product. The multiplication is

$$
(\alpha 1+x) \circ(\beta 1+y)=[\alpha \beta+(x, y)] 1+\alpha y+\beta x
$$

$\alpha, \beta$ scalars, $x, y$ in $V$ and $(x, y)$ their scalar product. Such an algebra is said to be of type $D$.

If $n \geqq 3$, there are 4 types: $A, B, C, E$. Types $A, B, C$ are special, while type $E$ is the exceptional algebra described above. To each of the types $A, B, C$ (and also to $D$ ) corresponds an associative algebra $\mathfrak{u}$ such that if the corresponding Jordan algebra is contained in an algebra $\mathfrak{A}_{j}, \mathfrak{A}$ associative, then the associative subalgebra of $\mathfrak{U}$ generated by $\mathfrak{\Im}$ (enveloping algebra of $\mathfrak{\Im}$ ) is a homomorphic image of $\mathfrak{U}$.

Type $A: \quad \mathfrak{F}=\mathfrak{A}_{j}, \mathfrak{A}$ the associative algebra of all $n \times n$ matrices over the base field, $n \geqq 3 . \quad \mathfrak{U}=\mathfrak{A} \oplus \mathfrak{A}$

Type $B: \Im$ is the algebra of all $n \times n$ symmetric matrices, $n \geqq 3$. $\mathfrak{U}=\mathfrak{U}(\mathfrak{U}$ as for type $A$ ).

Type $C: \Im$ is the algebra of all $2 n \times 2 n$ symplectic-symmetric 
matrices, isomorphic to the set of all self-adjoint linear transformations on a vector space $V$ with non-degenerate skew-symmetric scalar product. $\mathfrak{u}$ is the algebra of all linear transformations on $V$.

Type $D: \mathfrak{U}$ is the Clifford algebra determined by the space $V$ and the inner product.

If the base field is not algebraically closed, then the algebras which become of type $A$ on extension of the base field are of two subtypes:

$A_{1}: \mathfrak{\Im}=H(\mathfrak{H}), \mathfrak{A}$ a simple algebra with involution such that the involution is not the identity automorphism on the center of $\mathfrak{A} . \quad \mathfrak{U}=\mathfrak{A}$.

$A_{2}: \quad \mathfrak{S}=\mathfrak{A}_{j}, \mathfrak{A}$ a central simple associative algebra.

$\mathfrak{U}=\mathfrak{A} \oplus \mathfrak{U}^{\prime}, \mathfrak{U}^{\prime}$ anti-isomorphic to $\mathfrak{A}$.

The algebras that become types $B$ or $C$ are of the form $\mathfrak{\Im}=H(\mathfrak{A})$, $\mathfrak{A}$ simple involutorial with the involution acting as the identity automorphism on the center. $\mathfrak{U}=\mathfrak{A}$.

Algebras of type $D$ over an arbitrary base field are as described above for an algebraically closed base field. $\mathfrak{U}=C$, the Clifford algebra.

Algebras of type $E$ need not be algebras of all $3 \times 3$ hermitian matrices if the base field is not algebraically closed, according to recent unpublished work of A. A. Albert.

Section 1. Operator Commutativity. We will consider Jordan algebras over fields of characteristic different from 2 ; this assumption on characteristic will be made throughout this paper.

The concept of Operator-Commutativity, introduced by Jordan, von Neumann and Wigner in [17], is the natural analogue of the concept of commutativity of two elements in an associative algebra, as some of the following propositions will show. Some of these results were proved for characteristic zero in [8].

Let $\mathfrak{\Im}$ be a Jordan algebra, $x$ and $y$ elements of $\Im$. Let $x \circ y$ denote their product and write $x^{2}$ for $\frac{1}{2}(x \circ x)$. We denote by $R_{x}$ the linear transformation $a \rightarrow a \circ x$ acting in $\Im$.

Definition. Two elements $x, y$ of $\mathfrak{\Im}$ operator-commute (we will write also: 0 -commute) if $R_{x} R_{y}=R_{y} R_{x}$.

The set of elements of $\mathfrak{\Im} o$-commuting with $x$ will be denoted by $\widetilde{c}(x)$. If $\Omega$ is a subalgebra of $\mathfrak{\Im}$, the set of elements of $\mathfrak{\Im}$ o-commuting with all elements of $\Re$ will be denoted by $\mathfrak{S}_{\Im}(\Re)$.

If $\mathfrak{A}$ is an associative algebra and $\mathfrak{B}$ an associative subalgebra, we will write $\mathfrak{E} \mathfrak{a}(\mathfrak{B})$ for the subalgebra of elements of $\mathfrak{A}$ commuting with the elements of $\mathfrak{B}$. $\quad \mathcal{S} \mathfrak{Y}(\mathfrak{U})$ is the center of $\mathfrak{A}$-its elements will be called "central".

The following example, due to McCoy [14], and Jacobson [8], shows that the set $\mathfrak{c} \Im(x)$ of elements of $\Im o$-commuting with an element $x$ of 
$\mathfrak{\Im}$ is not necessarily a subalgebra of $\mathfrak{\Im}$ : $\mathfrak{\Im}$ will be a special Jordan algebra (product $x \circ y=x y+y x$ ) consisting of $6 \times 6$ matrices whose coefficients are rational numbers, namely the Jordan algebra over the field of rational numbers generated by the following matrices $a, b, 1$ (using $e_{k l}$ to denote the matrix with 1 in the $k, l$ position and zeros elsewhere, $k, l=1, \ldots, 6): \quad a=e_{14}+e_{25}+2 e_{16}, \quad b=e_{42}+e_{53}+e_{65}$, and $1=e_{11}+\cdots+e_{68}$. Let $c=a b-b a$, then $c=e_{12}+e_{23}+e_{45}$ and $c^{2}=$ $e_{13} \neq 0$. Also $a c=c a, b c=c b$, so that $c$ commutes with every polynomial in $a$ and $b$. Consider now $\varsigma_{\Im}(a)$ : we claim $\varsigma_{\Im}(a)$ contains $b$ but not $b^{\prime}$. The equation $(a \circ x) \circ b=a \circ(x \circ b)$ becomes, on replacing $y \circ z$ by $y z+z y$, $[[a, b], x]=0$ where $[y, z]$ denotes $y z-z y$. But $[a, b]=c$ and $c$ clearly commutes with every element of the Jordan algebra generated by $a$ and $b$, so $b o$-commutes with $a$. We compute $\left[a, b^{2}\right]$ and show this element does not commute with every $x$ in $\mathfrak{\Im}$ :

$$
\begin{aligned}
& {\left[a, b^{2}\right]=[a, b] b+b[a, b]=b c+c b=2 b c=2[a, b] b} \\
& {\left[\left[a, b^{2}\right], a\right]=2[b c, a]=2[b, a] c+2 b[c, a]=-2[a, b] c=-2 c^{2} \text { as }[a, c]=0}
\end{aligned}
$$

but $c^{2} \neq 0$, and $2 c^{2} \neq 0$, thus $b^{2}$ is not in $\mathfrak{c}_{\Im}(a)$. We note also that $b$ does not $o$-commute with $a^{2}$ and that $[a, b]^{2} \neq 0$.

In the preceding example, $b$ did not $o$-commute with all elements of the subalgebra generated by $\alpha$ and 1 . One may ask whether $\mathbb{E}_{\Im}(\Re)$ is a subalgebra if $\Omega$ is a subalgebra. This is so in many cases, as the following propositions will show, and we conjecture that it is true in general. Some of the following results were proved in [8] for characteristic zero.

We say a finite dimensional Jordan algebra $\mathfrak{\Im}$ over a field $F$ is separable if it is semi-simple and the algebra $\Im \bigotimes_{F} E$ obtained by extending the base field to $E$ is also semi-simple for any extension field $E$ of $F$. We note at this time a few simple facts about the effect of field extension on centralizers : if $x$ and $y$ are elements of $\Im$, they $o$ commute in $\mathfrak{\Im}$ if and only if they $o$-commute in $\mathfrak{\Im} \otimes E$; also, since the equation $R_{a} R_{b}=R_{b} R_{a}$ expressing that two elements $a$ and $b o$-commute

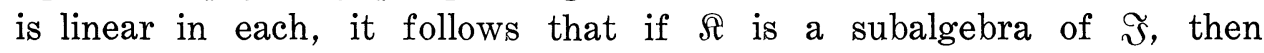
$\mathfrak{E}_{\Im} \otimes E(\Re \otimes E)=\mathfrak{C}_{\Im}(\Re) \otimes E$, and this allows us to extend the base field in many of our arguments.

Proposition 1.1. Let $\Im$ be any Jordan algebra (possibly infinite dimensional), $\Re$ a separable subalgebra. Then $\mathfrak{C}_{\Im}(\Omega)$ is a subalgebra of $\mathfrak{~}$.

Proof. First we show that if $e$ is an idempotent in $\mathfrak{F}$, then $\mathfrak{C}_{\mathfrak{F}}(e)$ is a subalgebra. Let $\mathfrak{\Im}=\mathfrak{\Im}_{0}+\mathfrak{Y}_{1 / 2}+\mathfrak{\Im}_{1}$ be the Peirce decomposition of $\mathfrak{\Im}$ relative to $e$, i.e. $\mathfrak{\Im}_{i}=\{x \in J \mid e \circ x=i x\}, i=0,1 / 2,1$. (We will also 
write $\mathfrak{\Im}_{e}(i)$ for $\left.\mathfrak{\Im}_{i}.\right) \mathfrak{\Im}_{0}$ and $\mathfrak{\Im}_{1}$ are subalgebras, and $\mathfrak{\Im}_{0} \mathfrak{\Im}_{1}=0, \mathfrak{\Im}_{0} \mathfrak{\Im}_{1 / 2} \subseteq \mathfrak{\Im}_{1 / 2}$, $\mathfrak{\Im}_{1} \mathfrak{\Im}_{1 / 2} \subseteq \mathfrak{\Im}_{1 / 2}$. From these relations it follows that $\mathfrak{c}_{\mathfrak{s}}(e)=\mathfrak{\Im}_{0}+\mathfrak{\Im}_{1}$ : let $x$ be in $\mathfrak{\Xi}_{\Im}(e)$, and let $x=x_{0}+x_{1 / 2}+x_{1}, x_{i}$ in $\Im_{i}$. Then $e \circ(e \circ x)=$ $x \circ(e \circ e)=x \circ e$, but $x \circ e=(1 / 2) x_{1 / 2}+x_{1}$, and $e \circ(x \circ e)=(1 / 4) x_{1 / 2}+x_{1}$, so $x_{1 / 2}=0$ and $x$ is in $\Im_{0}+\Im_{1}$. Conversely, let $x$ be in $\mathfrak{\Im}_{0}, z=z_{0}+z_{1 / 2}+z_{1}$ in $\Im$. $\quad x \circ(e \circ z)=x \circ\left[(1 / 2) z_{1 / 2}+z_{1}\right]=1 / 2\left(x \circ z_{1 / 2}\right)$, whereas $e \circ(x \circ z)=$ $e \circ\left(x \circ z_{0}+x \circ z_{1 / 2}\right)=1 / 2\left(x \circ z_{1 / 2}\right)$ since $x \circ z_{0}$ is in $\mathfrak{\Im}_{0}, x \circ z_{1 / 2}$ is in $\mathfrak{\Im}_{1 / 2}$; thus $x$ o-commutes with $e$, and similarly if $x$ is in $\mathfrak{J}_{1}$ it $o$-commutes with $e$. On extending the base field $F$ of $\mathfrak{\Im}$ to its algebraic closure, $\Re$ remains semi-simple, and we will conclude the proof by showing that a semisimple Jordan algebra over an algebraically closed base field has a basis consisting of idempotents-for if $e_{1}, \ldots, e_{n}$ are this basis, then $\mathfrak{C}_{\mathfrak{s}}(\Re)$ is the intersection of all the $\mathfrak{C}_{\mathfrak{s}}\left(e_{i}\right)$ and the latter are subalgebras. From the structure theory, it is known that $\Omega$ is a direct sum of simple algebras, and each simple algebra is either of degree one, i.e. of the form $F \cdot e, e$ an idempotent, or else is a vector space sum of algebras of degree two. An algebra of degree two has a basis of elements $e_{1}, e_{2}, x_{1}, \ldots, x_{n}$ where the $e_{i}$ are idempotents with $e_{1}+e_{2}=e$ being the identity element of the algebra, and $x_{i} \circ x_{i}=e$. Then $1 / 2\left(e+x_{i}\right)$ is also an idempotent, and $e_{1}, e_{2}, 1 / 2\left(e+x_{1}\right), \ldots, 1 / 2\left(e+x_{n}\right)$ is a basis consisting of idempotents, which we had to show.

The next proposition shows that for a large class of special Jordan algebras, including all that we will be concerned with in later sections, the set of elements o-commuting with the elements of a subalgebra is the same as the set of elements commuting with them in the associative multiplication. In particular, if $\mathfrak{\Im}=\mathfrak{U}$ is such an algebra, where $\mathfrak{A}$ is associative, and $\mathscr{R}=\mathfrak{B}_{j}$, where $\mathfrak{B}$ is an associative subalgebra of $\mathfrak{A}$, then $\mathfrak{\complement}_{\Im}(\Re)=\left[\mathfrak{C}_{\mathfrak{r}}(\mathfrak{B})\right]_{j}$.

Proposition 1.2. Let $\mathfrak{\Im}$ be a special Jordan algebra with enveloping associative algebra $\mathfrak{A}$, and assume $\mathfrak{A}$ has no central nilpotent elements

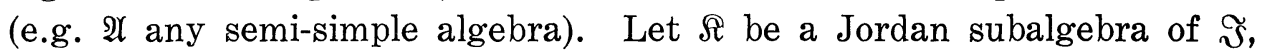
$y$ an element of $\mathfrak{\Im}$. Then $y$ is in $\mathfrak{c} \Im(\Re)$ if and only if $x y=y x$ for all $x \in \Re$. Thus $\mathbb{E} \Im(\Re)$ consists of all elements of $\mathfrak{\Im}$ commuting in the associative multiplication with the elements of $\Re$, and $\mathfrak{E}_{\mathfrak{S}}(\Re)$ is a subalgebra of $\mathfrak{\Im}$.

Proof. We make extensive use of the assumption that $2 \neq 0$. Also, we note that the equation $z\left(R_{x} R_{y}-R_{y} R_{x}\right)=0$ is equivalent to [[xy]z] $=0$, where $[a b]=a b-b a$. Let now $x \in \Re, y \in[(\Im)$. Since $\Re$ is a subalgebra, $x \circ x=2 x^{2} \in \Re$, and $x^{2} \in \Re$ so that $[[x y] z]=0$ and $\left[\left[x^{2} y\right] z\right]=0$ for all $z \in \mathfrak{\Im}$. Since $\mathfrak{A}$ is the enveloping algebra of $\mathfrak{\Im},[x y]$ and $\left[x^{2} y\right]$ are in the center of $\mathfrak{A}$. But $\left[x^{2} y\right]=[x y] x+x[x y]=2 x[x y]$ since $[x y]$ is 
in the center of $\mathfrak{A} .\left[x^{2} y\right] \in$ the center of $\mathfrak{A}$ implies that

$$
0=\left[y\left[x^{2} y\right]\right]=2[y,(x[x y])]=2[y x][x y]+0=-2[x y]^{2} .
$$

Thus $[x y]^{2}=0$, so by hypothesis on $\mathfrak{A},[x y]=0$. Conversely, if $[x y]=0$, then $[[x y] z]=0$ so that $x o$-commutes with $y$. The statements on $\widetilde{c}_{\mathfrak{s}}(\Re)$ now follow.

Let $\mathfrak{U}$ be a possibly non-associative algebra with multiplication denote by $x y$. We again introduce the new multiplication $x \circ y=$ $x y+y x$. Let $\mathfrak{B}$ be a subspace of $\mathfrak{A}$ closed under $x \circ y$, then we denote by $R_{y}$ the operation $x \rightarrow x \circ y=x R_{y}$ acting in $\mathfrak{B}$, where $x, y$ are elements of $\mathfrak{B}$. As before we say $x$ and $y$ o-commute if $R_{x} R_{y}=R_{y} R_{x}$. We can make a remark in case $\mathfrak{A}$ is a matrix algebra with canonical involution and $\mathfrak{B}$ is the set of self-adjoint elements (see [9]), i.e. $\mathfrak{A}$ is a matrix algebra $D_{n}$ over an algebra $D$ with identity 1 and involution $d \rightarrow \bar{d}$ in $D$, and $\mathfrak{A}$ has involution $a=\sum_{i, j} d_{i j} e_{i j} \rightarrow \sum_{i, j} \gamma_{j}^{-1} \bar{d}_{i j} \gamma_{i} e_{j i}$ where the $e_{i j}$ are matrix units, $\gamma_{1}=1, \gamma_{2}, \ldots, \gamma_{n}$ are self-adjoint elements of the nucleus of $D$ having inverses, and $n \geqq 2 ; \mathfrak{B}$ is the set of self-adjoint matrices, denoted by $H\left(D_{n}\right)$. Such algebras have been studied in [9], and include all simple Jordan algebras of degree greater than two over algebraically closed fields.

LEMma. Let $\mathfrak{A}=D_{n}$ be a matrix algebra with canonical involution, $\mathfrak{B}=H\left(D_{n}\right), x, y$ elements of $\mathfrak{B}$. If $x$ and $y$ o-commute, then $[x y]=d \cdot I$, where $d$ is a skew element of $D$, and $I$ is the unit matrix.

Proof. Since $x$ and $y$ are self-adjoint, $[x y]$ is skew. $\mathfrak{B}$ contains the elements $e_{i j}$ and $d[i, j]=d e_{i j}+\gamma_{j}^{-1} \bar{d}_{r_{i}} e_{j i}$ for $i \neq j$. Since $x$ and $y o-$ commute, we have $\left(e_{i i} \circ x\right) \circ y=\left(e_{i i} \circ y\right) \circ x$, which is equivalent to $\left[e_{i i}[x y]\right]$ $=0, i=1, \ldots, n$, since $e_{i i}$ is in the nucleus of $\mathfrak{A}$. The matrix [xy] thus has zeros off the main diagonal. The elements $1[i j]=e_{i j}+\gamma_{j}^{-1} \gamma_{i} e_{j i}$ are in the nucleus since the $\gamma_{i}$ and $e_{i j}$ are, so that

$$
\left[\left(e_{i j}+\gamma_{j}^{-1} \gamma_{i} e_{j i}\right),[x y]\right]=0, \quad i \neq j
$$

or

$$
e_{i j}[x y]+\gamma_{j}^{-1} \gamma_{i} e_{j i}[x y]=[x y] e_{i j}+[x y] \gamma_{j}^{-1} \gamma_{i} e_{j i} .
$$

Denote by $[x y]_{i j}$ the $i, j$ entry of $[x y]$ for $i, j=1, \ldots, n$. Then

$$
[x y]_{j j} e_{i j}+\gamma_{j}^{-1} \gamma_{i}[x y]_{i i} e_{j i}=[x y]_{i i} e_{i j}+[x y]_{j j} \gamma_{j}^{-1} \gamma_{i} e_{j i} \text { for } i \neq j .
$$

Since the coefficients of $e_{i j}$ in the above equation must be equal,

$$
[x y]_{j j}=[x y]_{i i}=d \text { for all } i, j \text {. }
$$

Thus $[x y]=d I, \bar{d}=-d$, which completes the proof.

From this lemma we can derive conditions for the centralizer of a 
subalgebra of $H\left(D_{n}\right)$ to consist of the matrices commuting with the matrices of the subalgebra. We denote by $Z$ the set of elements of $D$ commuting with every element of $D$, and by $N$ (nucleus) the set of elements of $D$ associating with every pair of elements of $D$.

Proposition 1.3. Let $\mathfrak{B}=H\left(D_{n}\right), n \geqq 2$.

(a) Let $n$ not be divisible by the characteristic of $F$. If $x$ and $y$ are two matrices of $\mathfrak{B}$ such that the coefficients of $x$ commute with those of $y$ and if $x$ and $y$-commute, then they commute. In particular if $x$ has coefficients in $Z$ and $y o$-commutes with $x$, then $x y=y x$.

(b) Let $\Re$ be a subalgebra of $H\left(D_{n}\right)$ such that every element of $\Omega$ has coefficients in $N$, the nucleus of $D$. If $Z$ contains no skew-elements whose squares are zero then $y$ is in $\mathfrak{E}_{\mathfrak{B}}(\Re)$ if and only if $x y=y x$ for all $x$ in $\mathscr{R}$, so $\mathfrak{C}_{\mathfrak{B}}(\Re)$ is a subalgebra. In particular, if $D$ is associative with no central skew elements of square zero, the conclusion holds for any subalgebra $\Re$ of $H\left(D_{n}\right)$. If $\mathfrak{B}$ is an exceptional simple Jordan algebra, then $D$ is a Cayley algebra and $Z=N=F \cdot 1, F$ the base field, so that the conclusion holds for subalgebras $\Re$ whose elements have coefficients in $F$.

Proof. (a) By the lemma, if $y$ o-commutes with $x$, then $[x y]=d I$, $d \in D, I=$ identity matrix. Let us now take the trace of the elements on each side of this equation. If the coefficients of $x$ commute with those of $y$, then $\operatorname{tr}(x y)=\operatorname{tr}(y x)$ so $\operatorname{tr}([x y])=0=\operatorname{tr}(d I)=n d$. Since $n d=0$ implies $d=0, x y=y x$.

(b) Let every $x$ in $\Omega$ have coefficients in $N$. Then $(x y) z=x(y z)$ for all $y, z \in D_{n}$. Let now $y \in\left(\mathcal{S}_{\mathfrak{B}}(\Omega)\right.$. Then $(x \circ z) \circ y=(y \circ z) \circ x$ for all $z$ in $\mathfrak{B}$, which is equivalent to: $[[x y] z]=0$ for all $z$ in $\mathfrak{B}$. $[x y]=d I$, so $d z_{i j}=z_{i j} d$ for each coefficient $z_{i j}$ of $z$. Since $n \geqq 2$, for any $a \in D$, there is a $z$ in $\mathfrak{B}$ with $z_{12}=a$. Thus $d$ is in $Z$. Since $x^{2}$ is in $\Re$ also, $\left[x^{2} y\right]=f I$ where $f$ is a skew element of $Z$. The calculation of Proposition 1.2 is still valid since $x$ and $x^{2}$ associate with any two elements of $\mathfrak{B}$, and we conclude that $d^{2}=0$ and so $d=0$, or $x y=y x$. The remaining statements of the proposition are now obvious.

Proposition 1.4. Let $\mathfrak{M}$ be an exceptional central simple Jordan algebra, and $\Re$ a separable subalgebra of $\mathfrak{M}$ containing the identity. Then $\left(\mathrm{Sm}_{(}(\Re)\right.$ is separable, and, if the base field is algebraically closed, $\mathfrak{M}$ can be represented as an $H\left(C_{3}\right)$ such that $\mathcal{C}_{\mathfrak{M}}(\Omega)$ consists of the matrices in $\mathfrak{M}$ commuting with those in $\Re$.

Proof. We first assume the base field $F$ algebraically closed, and $\Re$ semi-simple. $\mathfrak{M}$ is then of degree 3 , i.e. if $1=e_{1}+\cdots+e_{r}, e_{i}$ primitive mutually orthogonal idempotents in $\mathfrak{M}$, then $r=3$, and $\mathfrak{M}$ is 
a vector space $\operatorname{sum} \sum_{i, j} \mathfrak{M}_{i j}, i \leqq j, i, j=1,2,3$ where

$$
\begin{aligned}
& \mathfrak{M}_{i j}=\left\{x \in M \mid e_{i} \circ x=(1 / 2) x=e_{j} \circ x\right\} \text { for } i \neq j, \text { and } \\
& \qquad \mathfrak{M}_{i i}=\left\{x \in M \mid e_{i} \circ x=x\right\}=F e_{i} .
\end{aligned}
$$

These facts limit $\Re$ to a few possibilities :

1. $\Re$ not simple: then $\Omega=\Re_{1} \oplus \cdots \oplus \Re_{r}, \Omega_{i}$ simple and $r=2$ or 3 , since the identity element 1 of $\mathfrak{M}$ is also in $\Re$, and $1=u_{1}+\cdots+u_{r}$, $u_{i}$ the identities of $\Omega_{i}$.

(a) $r=3$. Then $u_{1}, u_{2}, u_{3}$ are primitive (in $\mathfrak{M}$ ) orthogonal idempotents and each $\Omega_{i}$ is of degree 1 so that we may take $e_{i}=u_{i}$. We now represent $\mathfrak{M}$ as $H\left(C_{3}\right)$ with $e_{i}=(1 / 2) e_{i i}$. We have $\Omega_{i}=F e_{i}$ and $\mathfrak{C M}(\Re)=\cap_{i} \mathfrak{S M}_{\mathfrak{M}}\left(e_{i}\right)$. But $\mathfrak{C M M}_{\mathfrak{M}}\left(e_{i}\right)=\mathfrak{M}_{i i}+\mathfrak{M}_{j j}+\mathfrak{M}_{j k}+\mathfrak{M}_{k k}$, where $j, k$ $\neq i$ since with respect to $e_{i}, \mathfrak{M}_{1}=\mathfrak{M}_{i i}$ and $\mathfrak{M}_{0}=\sum_{j, k \neq i} \mathfrak{M}_{j k}$. Hence $\mathfrak{S M}(\Re)=\mathfrak{M}_{11}+\mathfrak{M}_{22}+\mathfrak{M}_{33}=\Re . \quad$ It is clear that the matrices of $\mathfrak{S M}(\Re)$ commute with those of $\Omega$. Conversely, any matrix commuting with $e_{1}$, $e_{2}, e_{3}$ is a linear combination of $e_{1}, e_{2}, e_{3}$, and thus in $\widetilde{C}_{\mathfrak{M}}(\Omega)$.

(b) $r=2$. Then $\Omega=\Re_{1} \oplus \Omega_{2}$ and $\Re_{i}$ has identity $u_{i}$. Since $1=$ $u_{1}+u_{2}$, one of the $u_{i}$ must be primitive in $\mathfrak{M}$, say $u_{1}$, and the other one not: $u_{1}=e_{1}, u_{2}=e_{2}+e_{3}, e_{i}$ primitive orthogonal idempotents. Again write $\mathfrak{M}=H\left(C_{3}\right),(1 / 2) e_{i i}=e_{i}$. Here there are two cases

(i) $\Re_{2}$ is of degree one: $\Re_{2}=F u_{2}$. Since $\Re_{1}=F u_{1}$,

$$
\begin{aligned}
\mathfrak{C} \mathfrak{M}(\mathfrak{R}) & =\mathfrak{E M}_{\mathfrak{M}}\left(u_{1}\right) \cap \mathfrak{C} \mathfrak{M}\left(u_{2}\right) \\
& =\left(\mathfrak{M}_{11}+\mathfrak{M}_{22}+\mathfrak{M}_{23}+\mathfrak{M}_{33}\right) \cap\left(\mathfrak{M}_{11}+\mathfrak{M}_{22}+\mathfrak{M}_{23}+\mathfrak{M}_{33}\right) \\
& =\mathfrak{M}_{11}+\mathfrak{M}_{22}+\mathfrak{M}_{23}+\mathfrak{M}_{33} .
\end{aligned}
$$

But $\mathfrak{M}_{22}+\mathfrak{M}_{23}+\mathfrak{M}_{33}$ is a simple Jordan algebra $\mathfrak{M}_{11}^{\prime}$ of degree two, so that $\mathfrak{C M}_{\mathfrak{M}}(\Re)=\mathfrak{M}_{11} \oplus \mathfrak{M}_{11}^{\prime}$ is semi-simple. The matrices of $\mathfrak{C}_{\mathfrak{M}}(\mathfrak{\Omega})$ are evidently just those matrices commuting with $e_{1}$ and $e_{2}+e_{3}$ and therefore with the matrices of $\Omega$.

(ii) $\Omega_{2}$ is of degree two: $u_{2}=e_{2}+e_{3}$ where both $e_{2}$ and $e_{3}$ are in $\Omega_{2}$. Then $\Omega_{2}$ is a simple Jordan algebra of degree two, and so contains an element $a$ with $a \circ a=4\left(e_{2}+e_{3}\right), a \in \mathfrak{M}_{23}$. Since $e_{1}, e_{2}, e_{3}$ all belong to $\Re, \mathfrak{C M}(\Re) \subseteq F e_{1}+F e_{2}+F e_{3}$. Also, since $\mathfrak{C M}_{\left(e_{1}\right)}=\mathfrak{M}_{11}+\mathfrak{M}_{22}+\mathfrak{M}_{23}+\mathfrak{M}_{33}$ $\supseteqq \Re$, we see that $F e_{1} o$-commutes with $\Re$, i.e. $F e_{1} \subseteq C_{M}(\Omega)$. Let now $\alpha e_{2}+\beta e_{3}$ belong to $\operatorname{Cm}(\Omega)$ : then this element $o$-commutes with $a$, so

$$
\begin{gathered}
{\left[e_{2} \circ\left(\alpha e_{2}+\beta e_{3}\right)\right] \circ a=\alpha e_{2} \circ a=(1 / 2) \alpha a=\left(e_{2} \circ \alpha\right) \circ\left(\alpha e_{2}+\beta e_{3}\right)} \\
=(1 / 2) a \circ\left(\alpha e_{2}+\beta e_{3}\right)=(1 / 4)(\alpha+\beta) a .
\end{gathered}
$$

Thus $\alpha=\beta$, i.e. $\left(\mathcal{M M}_{(}(\Re) \subseteq F e_{1}+F\left(e_{2}+e_{3}\right)\right.$. On the other hand, $\left(e_{2}+e_{3}\right)$ $\in \mathfrak{S M}_{\mathfrak{M}}(\Re)$ since $\Re \subseteq \mathfrak{M}_{11}+\mathfrak{M}_{11}^{\prime}$, so $\widetilde{S}(\Re)=F e_{1}+F\left(e_{2}+e_{3}\right)$, and evidently these matrices commute with the matrices in $\Omega$. Conversely, let $x=\sum_{i \leqq j} x_{i j}, x_{i j} \in \mathfrak{M}_{i j}$, commute with the matrices of $\Re_{\text {. Since }} e_{1}, e_{2}, e_{3}$ 
are in $\Omega, x=\alpha e_{1}+\beta e_{2}+\gamma e_{3}$. Letting $e_{i j}, i \neq j$ denote the matrix units, the element $a$ of $\Omega$ can be written as $a=d e_{23}+\bar{d} e_{32}, d$ an element of $C$, and $a \circ a=4\left(d \bar{d} e_{2}+\bar{d} d e_{3}\right)=4\left(e_{2}+e_{3}\right)$, thus $d \bar{d}=\overline{d d}=1$.

$$
\begin{aligned}
& a x=\left(d e_{23}+\bar{d} e_{32}\right)\left(\alpha e_{1}+\beta e_{2}+\gamma e_{3}\right)=(1 / 2)\left(d \gamma e_{23}+\bar{d} \beta e_{32}\right) ; \alpha, \beta, \gamma \in F . \\
& x a=\left(\alpha e_{1}+\beta e_{2}+\gamma e_{3}\right)\left(d e_{23}+\bar{d} e_{32}\right)=(1 / 2)\left(\beta d e_{23}+\bar{\gamma} d e_{32}\right) .
\end{aligned}
$$

Since we are supposing $a x=x a$, we have $\beta d=d \gamma$, but $d \gamma=\gamma d$ as $\gamma \in F$, so $\beta=\gamma$, i.e. $x=\alpha e_{1}+\beta\left(e_{2}+e_{3}\right)$ and $x$ is in $\operatorname{Cs}(\Omega)$.

2. $\Omega$ is simple; then $\Omega$ has degree one, two, or three

(a) $\Omega$ has degree one: $\Omega=F \cdot 1$. Then $\left(\mathcal{S M}_{(2)}(\Re)=\mathfrak{M}\right.$, and the matrices of $\mathfrak{M}$ commuting with those of $\Re$ are just the elements of $(\mathfrak{M}(\Omega)$.

(b) $\Omega$ has degree two: we show this is impossible. For let $1=$ $u_{1}+u_{2}, u_{i}$ primitive idempotents in $\Omega$. Then, as in case $1 b$, we may assume $u_{1}$ is primitive in $\mathfrak{M}$ and $u_{2}$ not, so that $u_{1}=e_{1}, u_{2}=e_{2}+e_{3}$. Since $\Re$ is simple of degree two, it contains an element $x$ such that $x \circ x=2 x^{2}=u_{1}+u_{2}$ and $u_{1} \circ x=(1 / 2) x=u_{2} \circ x$. Let $x=\sum_{i \leqq j} x_{i j}, x_{i j}$ in $\mathfrak{M}_{i j}$. Since $e_{1} \circ x=(1 / 2) x, \quad x=x_{12}+x_{13}$, therefore $x \circ x=x_{12} \circ x_{12}+$ $x_{13} \circ x_{13}+2 x_{12} \circ x_{13}$. But

$$
x_{12} \circ x_{12}=\lambda_{1}\left(e_{1}+e_{2}\right), x_{13} \circ x_{13}=\lambda_{2}\left(e_{1}+e_{3}\right), \lambda_{i} \in F, x_{12} \circ x_{13} \in \mathfrak{M}_{23}
$$

so $x \circ x=\left(\lambda_{1}+\lambda_{2}\right) e_{1}+\lambda_{1} e_{2}+\lambda_{2} e_{3}+2 x_{12} \circ x_{13}=u_{1}+u_{2}=e_{1}+e_{2}+e_{3}$. Since $\mathfrak{M}$ is a direct sum of the $\mathfrak{M}_{i j}, 2 x_{12} \circ x_{13}=0, \lambda_{1}+\lambda_{2}=1, \lambda_{1}=1, \lambda_{2}=1$, a contradiction.

(c) $\Omega$ has degree three: Then $\Re$ contains idempotents $e_{1}, e_{2}, e_{3}$ and the subspaces $\Re_{i j}=\Re \cap \mathfrak{M}_{i j}$ are all non-zero. $\Re$ contains the subalgebra $\Re_{11}+\Re_{22}+\Omega_{23}+\Re_{33}$ of the type considered in $1 \mathrm{~b}$ (ii), and the centralizer of this subalgebra, as well as the set of matrices commuting with its elements, is $F e_{1}+F\left(e_{2}+e_{3}\right)$. Arguing the same way but replacing the index 1 by 2 and then 3 , we see that $\widetilde{M}(\Re)=F\left(e_{1}+e_{2}+e_{3}\right)=F \cdot 1$, and that any matrix commuting with the elements of $\Re$ is in $F \cdot 1$. Conversely, the matrices in $F \cdot 1$ obviously commute with those in $\Re$. We have shown that in each case $\widetilde{C}(\Omega)$ is semi-simple.

If now $F$ is not necessarily algebraically closed, the centralizer of $\Re$ in $\mathfrak{M}$ remains semi-simple on extending $F$ to its algebraic closure, so that $\left(\mho_{M}(\Re)\right.$ is separable. Also, in the algebraically closed case, we have shown that for every $\Omega$ there is a matrix representation of $\mathfrak{M}$ such that the elements of $\widetilde{M}(\Re)$ are represented by the matrices commuting with the matrices representing the elements of $\Omega$. This concludes the proof.

2. Subalgebras Generated by a Single Element. In this section we study the centralizer and double centralizer in a central simple Jordan 
algebra of the subalgebra generated by one element, and generalize to Jordan algebras some of the known results on simple associative algebras contained in, say, [6], Chapter 3.

If $x$ is an element of $\mathfrak{\Im}$, we denote by $(x)$ the subalgebra generated by $x$ and 1 . The following facts are known about $\mathfrak{s} \Im((x))$ if $\mathfrak{s}=\mathfrak{A}_{j}$ and $\mathfrak{A}$ is the associative algebra of all $n \times n$ matrices over a field $F$ :

1. Let $x$ have invariant factors $\delta_{1}, \cdots, \delta_{r}$ of respective degrees $d_{1} \geqq d_{2} \geqq \cdots \geqq d_{r}$. Let $(F[\lambda])_{r}$ be the algebra of all $r \times r$ matrices with coefficients polynomials in an indeterminate $\lambda,(\delta)$ the matrix diag $\left(\delta_{1}(\lambda), \cdots, \delta_{r}(\lambda)\right)$ and $\mathfrak{B}$ the subalgebra of matrices $(\alpha)$ in $(F[\lambda])_{r}$ such that $(\alpha)^{\prime}(\delta)=(\delta)(\beta)$ for some $(\beta)$ in $(F[\lambda])_{r}$, which condition we will also write as $(\delta)^{-1}(\alpha)^{\prime}(\delta)=(\beta) \in(F[\lambda])_{r},(\alpha)^{\prime}$ denoting the transpose of $(\alpha)$. Let $\Re$ be the ideal in $\mathfrak{B}$ of matrices of the form $(\beta)(\delta)$. Then $\widetilde{S}_{\mathfrak{a}}((x))$ is isomorphic to $\mathfrak{B} / \mathfrak{R}$, so that by the results of section $1, \quad \mathfrak{s} \Im((x))$ is isomorphic to $\mathfrak{B}_{j} / \mathfrak{R}_{j}$. From 1. easily follows the following theorem of Frobenius :

2. Let $x$ be as above with invariant factor degrees $d_{1} \geqq \cdots \geqq d_{r}$. Then $\mathcal{E}_{\mathfrak{a}}((x))$ is of dimension $\sum_{k=0}^{r-1}(2 k+1) d_{k+1}$.

3. Finally, $\operatorname{c} \mathfrak{x}\left(\operatorname{cr}_{\mathfrak{x}}((x))\right)=(x)$.

We will give appropriate generalizations of these results for Jordan algebras. The method will be to examine one by one the various types of simple algebras. In this way we will obtain some information on each of the various types of simple Jordan algebras, but it would also be interesting to have a general method which works for all the algebras at once.

Since all the special algebras $\Im$ that occur will have semi-simple enveloping associative algebras, we will be able to apply Proposition 1.2 to obtain that $\mathfrak{s}_{\Im}((x))$ is just the set of elements of $\mathfrak{\Im}$ commuting with $x$. Proposition $1.3 \mathrm{~b}$ will be used in a similar way for the exceptional algebras.

In most proofs the key case will be that of a nilpotent element $x$, and the reduction to that case will be made by using a special case of the decomposition of an associative algebra over a perfect field into a direct sum of a semi-simple algebra and the radical: if we consider the associative commutative subalgebra $(x)$ of $\Im$ and decompose it, then we can write $x=s+n, s$ a linear combination of orthogonal idempotents $e_{i}$ in $(x)$ and $n$ a sum of nilpotent elements $n_{i}$ in $(x)$ such that $e_{i} n_{i}=n_{i}$, $e_{j} n_{i}=0$ for $j \neq i$.

The known theorems about the algebra of all linear transformations quoted above are best proved by considering the vector space $V$ on which the given transformation $x$ acts as a module over a principal ideal 
ring and decomposing it into a direct sum of cyclic modules. If we are interested in vector spaces with inner product we have to try to obtain orthogonal decompositions into cyclic subspaces. This is done in the first two lemmas (which are essentially known, see e.g. [13], last chapter, for algebraically closed fields, but hard to find in the literature in a form useful for us).

Let $V$ be a finite dimensional left vector space over an involutorial division ring $D$. We will use the letters $x, y, \cdots$ for elements of $V, \alpha$, $\beta, \cdots$ for scalars in $D, \alpha \rightarrow \alpha^{\prime}$ denoting the involution, and $A, N, \cdots$ for linear transformations. We will assume $V$ is a self-dual space, i.e. it has a non-degenerate scalar product $(x, y)$ which is either hermitian: $(y, x)=(x, y)^{\prime},(\alpha x, \beta y)=\alpha(x, y) \beta^{\prime}$, or else alternate: $(x, y)=-(y, x)$ and $D$ is a field with $\alpha^{\prime}=\alpha$. $V$ will be called hermitian or alternate, respectively. The characteristic is assumed to be different from two.

LEMma 2.1. Let $V$ be a finite dimensional self-dual hermitian space over $D$, and $F$ the subfield of self-adjoint elements of $D$. Let $A$ be $a$ self-adjoint linear transformation which is algebraic over $F$, i.e. $A$ satisfies a polynomial equation over $F$, and assume also that if $D \neq F$ then the roots of this equation lie in $F$. Then $V$ can be decomposed into a direct sum of mutually orthogonal non-isotropic spaces which are indecomposable cyclic for $A$. If $A$ is nilpotent, then each cyclic subspace has a basis of the form $\left\{x, x A, \cdots, x A^{n-1}\right\}$ such that $\left(x A^{i}, x A^{j}\right)=0$ if $i+j \neq n-1$, and $\left(x A^{i}, x A^{n-1-i}\right)=\mu$ for all $i, \mu$ a self-adjoint non-zero element of $D$.

Proof. If the minimum polynomial of $A$ over $F$ has distinct prime factors, then corresponding to these there exist mutually orthogonal idempotents $E_{i}$ with sum 1 which are polynomials in $A$ with coefficients in $F$, and so are self-adjoint. Then $V=\sum \oplus V E_{i}$, and $\left(V E_{i}, V E_{j}\right)=$ $\left(V E_{i} E_{j}, V E_{j}\right)=0$ for $i \neq j$. Thus the $V_{i}=V E_{i}$ are mutually orthogonal and so are themselves hermitian self-dual spaces, and the linear transformation induced by $A$ on each of these satisfies a primary polynomial $p(\lambda)^{n}$ over $F$. Thus it is sufficient to assume the minimum polynomial of $A$ over $F$ is of the form $p(\lambda)^{n}$, and by assumption $p(\lambda)=\lambda-\alpha, \alpha$ in $F$, if $D$ is not equal to $F$. Moreover, if $p(\lambda)=\lambda-\alpha$, then it is sufficient to prove the first statement of the lemma for $A-\alpha 1$ instead of $A$, that is, we may assume $A$ nilpotent if $D$ is not $F$. In what follows we will often write $p$ or $p^{r}$ for $p(\lambda)$ or $p(\lambda)^{r}$, and if $m(\lambda)$ is a polynomial over $F$ in the indeterminate $\lambda$, we will write $z m(\lambda)$ or $z m$ for $z m(A)$, if $z$ is in $V$.

If $x$ is a vector of order $p^{r}$, that is, $x p(A)^{r}=0$ and $x m(A)=0 \mathrm{im}$ plies that $p^{r}$ divides $m$, then the vectors $x A^{0}=x, x A^{s}, 0<s<$ degree 
of $p^{r}$, form a basis for the cyclic subspace generated by $x$. This is well known if $D=F$, and if $D \neq F$, then $p(\lambda)=\lambda$ and so $x A^{r}=0$, $x A^{r-1} \neq 0$, and it is easy to check that the vectors $x, x A, \cdots, x A^{r-1}$ are linearly independent over $D$. We also note that $p(\lambda)^{n}$, the minimum polynomial of $A$ over $F$, is also the minimum polynomial of $A$ with coefficients in $D$, since if $p(\lambda)^{n}=\lambda^{n}$, the only monic factors of $p(\lambda)^{n}$ are the polynomials $\lambda^{r}$ which have coefficients in $F$. From now on all polynomials we will consider will be assumed to have coefficients in $F$.

Since $p(\lambda)^{n}$ is the minimum polynomial of $A$ over $D$, there is an $x$ in $V$ of order $p^{n}$. Let $U$ be the cyclic subspace generated by $x$, and suppose $U$ is isotropic. We show that this implies $\left(x p^{n-1}, x\right)=0$ : first let $D=F$, and let $x q(A)$ be a non-zero vector in the radical of $U$. Then $(x q(A), x t(A))=0=(x q(A) t(A), x)$ for all $t=t(\lambda)$. Since $x q(A) \neq 0$, there is a $t$ such that $x q(A) t(A)=x p(A)^{n-1}$. If $D \neq F$, let $z=\sum_{i=1}^{n-1} \gamma_{i} x A^{i}$ be non-zero and in the radical of $U$. Suppose $\gamma_{k} \neq 0$, and $\gamma_{j}=0$ for $j<k$. Then $0=\left(z, x A^{n-1-k}\right)=\gamma_{k}\left(x A^{k}, x A^{n-1-k}\right)=\gamma_{k}\left(x A^{n-1}, x\right)$. We now show that we can find a non-isotropic cyclic subspace $U$ of maximum order: once we have it, its orthogonal complement $U^{\perp}$ will also be invariant for $A$, and will satisfy the same hypotheses as $A$ but will have lower dimension, and we can use induction. Therefore, suppose $\left(x, x p^{n-1}\right)=0$. There exists $y$ in $V$ such that $\left(y, x p^{n-1}\right)=1=\left(y p^{n-1}, x\right)$. Thus $y p^{n-1} \neq 0$, and we may assume $\left(y, y p^{n-1}\right)=0$, otherwise $y$ generates the desired cyclic space. Consider now the cyclic subspace spanned by $x+y$ :

$$
\begin{aligned}
\left(x+y,(x+y) p^{n-1}\right) & =\left(x, x p^{n-1}\right)+\left(y, y p^{n-1}\right)+\left(x, y p^{n-1}\right)+\left(y, x p^{n-1}\right) \\
& =0+0+1+1=2 \neq 0 .
\end{aligned}
$$

Thus, in particular, $x+y$ has order $p^{n}$, and it generates a non-isotropic cyclic space of order $p^{n}$. This completes the proof of the first statement of the lemma.

Suppose $A$ is nilpotent and $V$ is cyclic of dimension $n$. Let $x, x A$, $\cdots, x A^{n-1}$ be a basis for $V$. Clearly $\left(x A^{n-1}, x A^{i}\right)=0$ for $i \geqq 1$, so we may assume $\left(x, x A^{n-1}\right)=\mu \neq 0$. If $n=1$, there is nothing to prove, so let $n \geqq 2$. Suppose $k$ is some integer such that $0 \leqq k<n-1$ and $\left(x, x A^{r}\right)=0$ for all $r$ satisfying $k<r<n-1$. We show that $x$ may be replaced by another cyclic generator $y$ such that $\left(y, y A^{r}\right)=0$ for $k-1<r<n-1$ : Let $y=x+\alpha x A^{n-1-k}, \alpha$ an element of $D$ to be specified shortly. For $k<r \leqq n-1, y A^{r}=\alpha x A^{n-1+r-k}+x A^{r}=x A^{r}$ since $A^{n}=0$; in particular $y A^{n-1} \neq 0$ so $y$ is a cyclic generator also. Further, for $k<r<n-1$,

$$
\begin{aligned}
\left(y, y A^{r}\right) & =\left(y, x A^{r}\right)=\left(x+\alpha x A^{n-1-k}, x A^{r}\right) \\
& =\left(x, x A^{r}\right)+\alpha\left(x, x A^{n-1+r-k}\right) \\
& =\left(x, x A^{r}\right) \text { since } r-k>1 .
\end{aligned}
$$


Finally,

$$
\begin{aligned}
\left(y, y A^{k}\right) & =\left(x+\alpha x A^{n-1-k}, x A^{k}+\alpha x A^{n-1}\right) \\
& =\left(x, x A^{k}\right)+\alpha\left(x, x A^{n-1}\right)+\left(x, x A^{n-1}\right) \alpha^{\prime}+\alpha\left(x A^{n-1-k}, x A^{n-1}\right) \alpha^{\prime} .
\end{aligned}
$$

The last term is zero since $n-1-k \geqq 1$, and $\left(x, x A^{n-1}\right)=\mu \neq 0$. Let $\alpha=-(1 / 2)\left(x, x A^{k}\right) \mu^{-1}$, then $\alpha\left(x, x A^{n-1}\right)=\alpha \mu=-(1 / 2)\left(x, x A^{k}\right)=\left(x, x A^{n-1}\right) \alpha^{\prime}$ since $\mu^{\prime}=\mu$, and so $\left(y, y A^{k}\right)=0$ also. This completes the proof.

This last type of basis can be used to give a relation between the index of the hermitian form and the index of nilpotency of a nilpotent self-adjoint linear transformation. Let $V$ have dimension $m$ and index $r$, that is, $r$ is the dimension of any maximal totally isotropic subspace, so that $m \geqq 2 r$. Let $N$ be nilpotent of index $n$, i.e. $N^{n}=0, N^{n-1} \neq 0$, and self-adjoint. Then : $n \leqq \operatorname{Min}(m, 2 r+1)$, (i.e. if $m=2 r$ then $n \leqq 2 r$ and if $m>2 r$ then $n \leqq 2 r+1$ ), and equality is achieved for some $N$ ). To see this, choose a set of vectors $x, x N, \cdots, x N^{n-1}$ satisfying $\left(x N^{j}, x N^{k}\right)$ $=0$ for $j+k \neq n-1,\left(x n^{j}, x N^{n-1-j}\right) \neq 0$. The vectors $x N^{j}$ for $j<$ $(n-1) / 2$ then span a totally isotropic subspace of dimension [n/2] (greatest integer $\leqq n / 2)$ so $[n / 2] \leqq r$ and $n \leqq 2 r+1$. The condition $n \leqq m$ is always satisfied. Conversely, let $V$ have index $r$. Then we can find vectors $x_{1}, \cdots, x_{r}$ and $y_{1}, \cdots, y_{r}$ spanning totally isotropic spaces respectively and satisfying $\left(x_{i}, y_{j}\right)=\gamma \delta_{i j}, \gamma$ any preassigned element of $D$. First assume $m>2 r$; then we can find a vector $z$ orthogonal to the space spanned by the $x_{i}$ and $y_{i}$ such that $(z, z) \neq 0$. We may assume $\left(x_{i}, y_{i}\right)=\gamma=(z, z)$ for all $i$. Now define a linear transformation $N$ as follows :

$$
\begin{aligned}
x_{1} N=x_{2}, x_{2} N=x_{3}, \cdots, x_{r-1} N & =x_{r}, x_{r} N=z, \\
z N & =y_{r}, y_{r} N=y_{r-1}, \cdots, y_{2} N=y_{1}, y_{1} N=0 .
\end{aligned}
$$

Let $U$ be the space spanned by the $x_{i}, y_{i}, z ; U$ is non-isotropic, so $U \oplus U^{\perp}=V, U^{\perp}$ denoting the orthogonal complement of $U$. Define $N$ to be zero on $U^{\perp}$. It is clear that $N$ is nilpotent of index $2 r+1$ with cyclic subspace $U$, and self-adjoint. If $m=2 r$, we merely omit $z$ and define $N$ by : $x_{1} N=x_{2}, \cdots, x_{r-1} N=x_{r}, x_{r} N=y_{r}, y_{r} N=y_{r-1}, \cdots, y_{2} N=$ $y_{1}, y_{1} N=0$, where $\left(x_{i}, y_{j}\right)=\delta_{i j}$.

Lemma 2.1 is a generalization of the result that a hermitian selfdual finite-dimensional space has an orthogonal basis, the one-dimensional orthogonal subspaces being replaced by cyclic subspaces of a self-adjoint linear transformation. We obtain an analogous generalization of a symplectic basis (i.e. $\left.\left(x_{i}, y_{j}\right)=\delta_{i j}=-\left(y_{j}, x_{i}\right),\left(x_{i}, x_{j}\right)=0=\left(y_{i}, y_{j}\right)\right)$ for a symplectic space:

Lemma 2.2. Let $V$ be a symplectic space and $A$ a self-adjoint linear transformation in $V$. Then $V$ can be decomposed into a direct sum of 
indecomposable cyclic subspaces $U_{i}, U_{i}^{\prime}$ such that $U_{i}$ and $U_{i}^{\prime}$ are isomorphic as $(A)$ modules and totally isotropic and $U_{i}+U_{i}^{\prime}$ is non-isotropic and orthogonal to $U_{j}+U_{j}^{\prime}$ if $i \neq j$. If $A$ is nilpotent and $U, U^{\prime}$ are a pair of isomorphic cyclic subspaces with $U+U^{\prime}$ non-isotropic, then we can find bases of the form $\left\{x, x A, \cdots, x A^{r-1}\right\},\left\{y, y A, \cdots, y A^{r-1}\right\}$ for $U, U^{\prime}$ respectively such that $\left(x A^{i}, y A^{r-1-i}\right)=1=-\left(y A^{i}, x A^{r-1-i}\right)$, and all other scalar products are zero.

Proof: As in the hermitian case, we can immediately reduce the proof to the case of a primary minimum polynomial $p(\lambda)^{n}$ for $A$. We note that every cyclic subspace is totally isotropic, for $\left(x A^{i}, x A^{\jmath}\right)=$ $-\left(x A^{j}, x A^{i}\right)$ since $V$ is symplectic, but $\left(x A^{i}, x A^{j}\right)=\left(x A^{j}, x A^{i}\right)$ since $A$ is self-adjoint, so $\left(x A^{i}, x A^{j}\right)=0$. This implies that if $U$ and $U^{\prime}$ are cyclic subspaces such that $U+U^{\prime}$ is non-isotropic, then their intersection contains just the zero vector, since any vector in the intersection is orthogonal to both $U$ and $U^{\prime}$ and so is orthogonal to $U+U^{\prime}$. As in the proof of Lemma 2.1, it will suffice to find isomorphic cyclic subspaces $U, U^{\prime}$ such that $U+U^{\prime}$ is non-isotropic (and therefore the sum is automatically direct).

Let $x$ be a vector of maximum order $p^{n}$ in $V$, and let $U$ be the cyclic subspace generated by $x . x p^{n-1}$ is not zero, so there exists $y$ in $V$ such that $\left(x p^{n-1}, y\right)=1$. Then also $\left(x, y p^{n-1}\right)=1$, so $y p^{n-1} \neq 0$ and $y$ also has order $p^{n}$. Let $U^{\prime}$ be the cyclic subspace generated by $y$ : then $U^{\prime}$ has order $p^{n}$ and so is isomorphic to $U$. Suppose $z$ is in the radical of $U+U^{\prime}$, and let $z=x f(A)+y g(A), f, g$ polynomials. If $f(A)$ is not zero, then $f$ is not divisible by $p^{n}$, and so there is a polynomial $h$ such that $f(A) h(A)=p(A)^{n-1}$. Then

$$
\begin{aligned}
0 & =(z, y h(A))=(z h(A), y)=\left(x p(A)^{n-1}+y g(A) h(A), y\right) \\
& =1 \text { since }\left(x p(A)^{n-1}, y\right)=1 \text { and }(y g(A) h(A), y)=0,
\end{aligned}
$$

a contradiction. Thus $f(A)=0$, and since $\left(y p(A)^{n-1}, x\right)=-1$, we obtain in the same way $g(A)=0$, so $z=0$. Thus $U+U^{\prime}$ is non-isotropic.

Now assume $A$ is nilpotent, and $U, U^{\prime}$ are isomorphic cyclic subspaces such that $U+U^{\prime}$ is non-isotropic. Let $x, x A, \cdots, x A^{r-1}$, and $y, y A, \cdots, y A^{r-1}$ be bases for $U, U^{\prime}$ respectively, and $A^{r}=0 . \quad\left(y A^{r-1}, x A^{i}\right)$ $=\left(y A^{r+i-1}, x\right)=0$ if $i>1$, and also $\left(y A^{r-1}, z\right)=0$ for every $z$ in $U^{\prime}$, thus we must have $\left(y A^{r-1}, x\right) \neq 0$ since otherwise $y A^{r-1}$ is in the radical of $U+U^{\prime}$. Replacing $x$ by a scalar multiple if necessary, we may assume $\left(x, y A^{r-1}\right)=1$.

Fix $k, 0 \leqq k<r-1$, and assume that $\left(x, y A^{j}\right)=0$ for $k<j<r-1$. Let $x^{\prime}=x+\alpha x A^{r-1-k}, \alpha$ a scalar to be specified shortly. Then $x^{\prime}$ is also a cyclic generator of $U$, and

$$
\left(x^{\prime}, y A^{r-1}\right)=\left(x, y A^{r-1}\right)+\alpha\left(x A^{r-1-k}, y A^{r-1}\right)=\left(x, y A^{r-1}\right)=1
$$


since $r-1-k \geqq 1$. Also, for $k<j<r-1$,

$$
\begin{aligned}
\left(x^{\prime}, y A^{j}\right)=\left(x, y A^{j}\right)+\alpha\left(x A^{r-1-k}, y A^{j}\right) & =\left(x, y A^{\jmath}\right)+\alpha\left(x, y A^{r-1+j-k}\right) \\
& =\left(x, y A^{\jmath}\right)
\end{aligned}
$$

since $j-k \geqq 1$, so $\left(x^{\prime}, y A^{j}\right)=0$ for $k<j<r-1$. Finally,

$$
\left(x^{\prime}, y A^{k}\right)=\left(x, y A^{k}\right)+\alpha\left(x, y A^{r-1}\right)=\left(x, y A^{k}\right)+\alpha .
$$

Let $\alpha=-\left(x, y A^{k}\right)$, then $\left(x^{\prime}, y A^{k}\right)=0$ also. Proceeding in this way we can obtain a cyclic generator $x^{\prime \prime}$ of $U$ such that

$$
\left(x^{\prime \prime}, y A^{n-1}\right)=1=-\left(y, x^{\prime \prime} A^{n-1}\right) \text {, and }\left(x^{\prime \prime}, y A^{j}\right)=0 \text { for } j \neq n-1 .
$$

This completes the proof.

The above lemma has several immediate consequences:

1. The invariant factors $\delta_{1}, \cdots, \delta_{r}$ of a self-adjoint linear transformation in a symplectic space come in pairs : $\delta_{1}=\delta_{2}, \cdots, \delta_{r-1}=\delta_{r}$ (and $r$ is even).

2. If $V$ has dimension $m$ there is a self-adjoint nilpotent linear transformation of index of nilpotency $m$.

3. If the base field is algebraically closed, two self-adjoint linear transformations are conjugate by an isometry of $V$ if and only if they have the same invariant factors.

The last statement, 3., is also valid for a space $V$ with symmetric scalar product over an algebraically closed field. This follows easily from Lemma 2.1.

In order to extend the theorem of Frobenius mentioned above to arbitrary central simple Jordan algebras, we have to define now invariant factor degrees, and preferably also invariant factor polynomials, for an element $x$ of such a Jordan algebra. For characteristic zero, there is a general method for doing this, due to Professor Jacobson (unpublished), based on Lie algebra methods: it is proved that if $x$ is a nilpotent element, then there exists another nilpotent element $y$ such that $x, y$ and the identity element of $\mathfrak{\Im}$ generate a semisimple subalgebra which is a direct sum of simple algebras $H_{i}$, where $H_{i}$ is the Jordan algebra of all self-adjoint linear transformations in a vector space of dimension $n_{i}$ with non-degenerate symmetric scalar product of maximal index, and if $n_{1} \geqq n_{2} \geqq \cdots \geqq n_{r}$, then $n_{1}$ is the index of nilpotency of $x$. It is then natural to define $\delta_{i}(\lambda)=\lambda^{n_{i}}, d_{i}=n_{i}$. We shall use the full statement of the above theorem only for the exceptional Jordan algebra over an algebraically closed field. In this case of the exceptional algebra the theorem has also been proved for characteristic $p \neq 2$ or 3 , based on (unpublished) work of Professor Jacobson on the representation theory of a simple 3-dimensional Lie algebra, so that we have to assume characteristic not 2 or 3 for the exceptional Jordan algebra in Theorems 2.2, 2.4. 
To avoid the difficulties of Lie algebra arguments in characteristic $p$, we will define the $d_{i}$ differently, and will use the above theorem of Professor Jacobson only in some of the proofs for the exceptional algebra.

Definition of the $d_{i}$ : Let $\mathfrak{\Im}$ be central simple, and extend the base field to its algebraic closure. If $\mathfrak{\Im}$ becomes the algebra of all $n \times n$ symmetric matrices (Type $B$ ) or all $n \times n$ matrices, Type $A$, take the usual definition of $d_{i}$, $\delta_{i}$ for the $n \times n$ matrix $x$. For Type $C$, the "symplectic-symmetric" matrix $x$ has invariant factors equal in pairs, and we take for $\delta_{i}$ one member of each pair, and for $d_{i}$ the degree of $\delta_{i}$. For type $D$, the element $x$ satisfies a minimum polynomial $\mu$ of degree one or two; let $\delta_{2}=\mu=\delta_{1}, d_{1}=d_{2}=1$ if $\mu$ is of degree one, and $\delta_{1}=\mu, \delta_{2}=0, d_{1}=2, d_{2}=0$ if $\mu$ is of degree two. Finally, if $\Im$ is an exceptional algebra, every element satisfies a minimum polynomial of degree at most $3([5])$ : set $d_{1}=3, d_{2}=d_{1}=0$, or $d_{1}=2, d_{2}=1, d_{3}=0$, or $d_{1}=d_{2}=d_{3}=1$ according as the degree is 3,2 , or 1 ; set $\delta_{1}=$ minimum polynomial of $x$. Finally, if $\mathfrak{\Im}$ is of degree one, then $\mathfrak{\Im}=F \cdot 1$, and we can set $d_{1}=1, d_{i}=0$ for $i>1$. In the following proofs the case of $\mathfrak{F}$ of degree one will not be mentioned because of its triviality.

The $d_{i}, \delta_{i}$ as defined above have the following properties:

(a) $\delta_{1}=$ minimum polynomial of $x$ in $\mathfrak{F} . d_{i} \geqq d_{i+1}$ and $\delta_{i+1}$ divides $\delta_{i}$ whenever defined.

(b) $d_{1} \leqq$ degree of $\mathfrak{\Im}$ (defined as the maximum number of orthogonal idempotents in a decomposition of the identity when the base field is extended to its algebraic closure). $\quad \sum_{i} d_{i}=$ degree of $\Im$.

(c) If $\mathfrak{\Im}$ is special (Types $A-D$ ) and the base field is algebraically closed, two elements are taken into one another by an automorphism of $\Im$ leaving the center fixed if and only if they have the same invariant factors. The same thing is true for $\mathfrak{\Im}$ an exceptional algebra, at least for characteristic zero, as will become apparent from some of the later proofs. However, we will not use this, and so do not give the details of a proof.

The next theorem describes the structure of $\mathfrak{s} \Im((x))$ if $\mathfrak{\Im}$ is central simple over an algebraically closed field and special. The exceptional algebra is studied during the proof of the succeeding theorem, but we cannot give a simple statement for it.

THEOREM 2.1. (a) Let $\mathfrak{\Im}$ be the Jordan algebra of all $n \times n$ symmetric matrices over an algebraically closed field $F, N$ a nilpotent element of $\Im$ with invariant factors $\delta_{1}, \delta_{2}, \cdots, \delta_{r}$ where $\delta_{i+1}$ divides $\delta_{i}$. Let $(F[\lambda])_{\text {r }}$ be the algebra of all $r \times r$ matrices with coefficients polynomials in the indeterminate $\lambda$, $\Omega$ the subalgebra of matrices $(\beta)=\left(\beta_{i j}(\lambda)\right)$ satisfying $(\delta)^{-1}(\beta)^{\prime}(\delta)=(\beta)$ where $(\delta)=\operatorname{diag}\left(\delta_{1}, \cdots, \delta_{r}\right)$ and $(\beta)^{\prime}$ is the transpose of $\beta$, and $\Re$ the ideal in $\Re$ of matrices $(\beta)$ of the form $(\beta)=(\alpha)(\delta)$ for some 
$(\alpha)$ in $(F[\lambda])_{r}$. Then $\complement_{\Im}((N))$ is isomorphic to $\Re / \Re$. If $A$ is an arbitrary

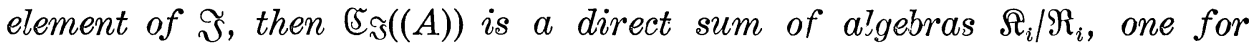
each characteristic root. If $\Im$ consists of all hermitian matrices over an arbitrary field and $N$ is a nilpotent element, then ${ } \Im(N)$ is as above with $\delta_{i}$ replaced by $\alpha_{i} \delta_{i}, \alpha_{i}$ a self-adjoint scalar, and $(\beta)^{\prime}$ the conjugate transpose of $(\beta)$.

(b) Let $\mathfrak{\Im}$ be the Jordan algebra of all $n \times n$ symplectic-symmetric matrices over an arbitrary field, $N$ a nilpotent element with invariant factors $\delta_{1}, \cdots, \delta_{r}$ in $\mathfrak{\Im}$ (i.e. the $\delta_{i}$ are every other one of the ordinary invariant factors). Then $\mathfrak{C}_{\Im}((N))$ is isomorphic to $\Re / \Re$ where $\Re$ is the subalgebra of $(F[\lambda])_{2 r}$ of matrices $(\beta)$ satisfying $(\delta)^{-1}(\beta)^{\prime}(\delta)=(\beta),(\delta)=$ $\delta_{1}\left(e_{12}-e_{21}\right)+\delta_{2}\left(e_{34}-e_{43}\right)+\cdots+\delta_{r}\left(e_{2 r-1,2 r}-e_{2 r, 2 r-1}\right)\left(e_{i j}\right.$ matrix units in $\left.(F[\lambda])_{2 r}\right)$, and $\Re$ is the ideal in $\Re$ of matrices of the form $(\beta)=(\alpha)(\delta)$.

(c) Let $\Im$ be the algebra $\left(F_{n}\right)_{j}$ of all $n \times n$ matrices over $F ; A$ an element of $\mathfrak{\Im}$ with invariant fastors $\delta_{1}, \cdots, \delta_{r}$. Let $\Omega$ be the subalgebra of $(F[\lambda])_{r}$ of all $(\beta)$ such that $(\delta)^{-1}(\beta)^{\prime}(\delta)$ is in $(F[\lambda])_{r},(\delta)$ as in (a), $\Re$ the ideal of $(\beta)$ of the form $(\beta)=(\alpha)(\delta),(\alpha)$ in $(F[\lambda])_{r}$. Then $\mathfrak{S}_{\mathfrak{s}}((A))$ is isomorphic to $\Re / \Re$.

(d) Let $\Im$ be central simple of Type D. Then $\mathfrak{\complement} \Im((x))=(x)$ if $x$ is not in the center of $\mathfrak{\Im}$, and $\mathfrak{\complement} \Im((x))=\mathfrak{\Im}$ otherwise.

Proof. (a) We take $\mathfrak{\Im}$ to be the set of all self-adjoint linear transformations in a hermitian self-dual space $V$, and $N$ a nilpotent element of $\mathfrak{\Im}$. Let $x_{1}, \cdots, x_{r}$ generate cyclic non-isotropic mutually orthogonal subspaces of orders $d_{i}=$ degree of $\delta_{i}, i=1, \cdots, r$ and satisfy $\left(x_{i}, x_{i} N^{j-1}\right)=\alpha_{i} \neq 0$ if $j=d_{i}-1,\left(x_{i}, x_{i} N^{j-1}\right)=0$ otherwise.

Let $B$ be any linear transformation commuting with $N$ and write $x_{i} B=\sum_{j=1}^{r} x_{j} \beta_{i j}(N), \beta_{i j}$ polynomials. Since $x_{i} \delta_{i}(N)=0, \delta_{i} \beta_{i j} \equiv 0\left(\bmod \delta_{j}\right)$. Also, since $B N=N B,\left(x_{i} N^{p} B, x_{j} N^{q}\right)=\left(x_{i} N^{p+q} B, x_{j}\right)$, so that $B$ is selfadjoint if and only if $\left(x_{i} N^{k} B, x_{j}\right)=\left(x_{i} N^{k}, x_{j} B\right)$ for all $i, j, k$.

Now fix $i, j, 1 \leqq i \leqq j \leqq r$ and let

$$
\begin{aligned}
& \beta_{i j}(\lambda)=\mu_{0}+\mu_{1} \lambda+\cdots+\mu_{p-1} \lambda^{p-1}, \quad p=d_{j} \\
& \beta_{3 i}(\lambda)=\nu_{0}+\nu_{1} \lambda+\cdots+\nu_{q-1} \lambda^{q-1}, \quad q=d_{i} \text {. }
\end{aligned}
$$

Since $\left(x_{j}, x_{j} N^{p}\right)=\alpha_{j},\left(x_{i}, x_{i} N^{q}\right)=\alpha_{i}$, and $\left(x_{i}, x_{i} N^{k}\right)=0$ for $k \neq q$, we have the condition:

$$
\begin{aligned}
& \left(x_{i} N^{k} B, x_{j}\right)=\left(x_{i} B N^{k}, x_{j}\right)=\mu_{p-1-k}\left(x_{j} N^{p-1}, x_{j}\right)=\alpha_{j} \mu_{p-1-k} \\
& \left(x_{i} N^{k}, x_{j} B\right)=\nu_{q-1-k}^{\prime}\left(x_{i} N^{q-1}, x_{i}\right)=\alpha_{i} \nu_{q-1-k}^{\prime}
\end{aligned}
$$

for all $k$, that is $\alpha_{i}^{-1} \mu_{p-1-k}=\alpha_{j}^{-1} \nu_{q-1-k}^{\prime}$ or $\alpha_{i}^{-1} \beta_{i j}(\lambda)=\lambda^{\left(a_{j}-a_{i}\right)} \alpha_{j}^{-1} \beta_{j i}(\lambda)^{\prime}$ 


$$
\beta_{i j}(\lambda)=\left[\alpha_{i}^{-1} \delta_{i}(\lambda)\right]^{-1} \beta_{j i}(\lambda)^{\prime}\left(\alpha_{j}^{-1} \delta_{j}(\lambda)\right)
$$

$\beta_{j i}(\lambda)^{\prime}$ denoting the conjugate of $\beta_{i i}(\lambda)$.

Since $\delta_{i}(\lambda) \beta_{i j}(\lambda) \equiv 0\left(\bmod \delta_{j}(\lambda)\right)$ is a consequence of the condition $(1)$, every matrix $(\beta)$ satisfying the above condition (1) defines a $B$ in $\mathfrak{c}_{\Im}((N))$ if we define for all $i, k x_{i} N^{k} B=\sum_{j} x_{j} \beta_{i j}(N) N^{k}$, and conversely every $B$ in $\mathfrak{E}_{\Im}(N)$ gives rise to such a matrix. Since $\delta_{j}$ is the order of $x_{j}$, it is clear that $B$ is zero if and only if $\beta_{i j} \equiv 0\left(\bmod \delta_{j}\right)$. This proves the isomorphism for $N$ nilpotent. If $A$ is an arbitrary element of $\Im$, in the symmetric case, and all the characteristic values $\lambda_{i}$ of $A$ lie in $F$, then we can write $A=\sum_{i} \lambda_{i} E_{i}+N_{i}$, the $E_{i}, N_{i}$ being the idempotent and nilpotent elements of $A$. Set $V_{i}=V E_{i}$, then $\Im_{\Im}(A)$ is the direct sum of $\mathfrak{s}_{\Im_{i}}\left(\left(N_{i}\right)\right)$, $\mathfrak{\Im}_{i}$ the symmetric linear transformations on $V_{i}$. If $F$ is algebraically closed, we may assume $\alpha_{i}=1$ for $V$ symmetric.

(b) We denote by $A$ the nilpotent symplectic-symmetric matrix under consideration. Regarding the elements of $\mathfrak{F}$ as self-adjoint linear transformations in $V$, we can choose a canonical basis $\left\{x_{i} A^{k}, y_{i} A^{k}\right\}$ as in Lemma 2.2: $\left(x_{i} A^{k}, y_{i}\right)=1=-\left(y_{i} A^{k}, x_{i}\right)$ if $k=d_{i}-1$, all other scalar products are zero. Let $B$ be a linear transformation in $V$ commuting with $A$, and let

Then

$$
\begin{aligned}
& x_{i} B=\sum_{j} x_{j} \varphi_{i j}(A)+y_{j} \psi_{i j}(A) \\
& y_{i} B=\sum_{j} x_{j} \eta_{i j}(A)+y_{j} \rho_{i j}(A) .
\end{aligned}
$$

$$
\begin{aligned}
& \left(x_{i} A^{k} B, y_{j}\right)=\left(x_{j} \varphi_{i j}(A) A^{k}, y_{j}\right) \\
& \left(x_{i} A^{k}, y_{j} B\right)=\left(x_{i} A^{k}, y_{i} o_{j i}(A)\right)=\left(x_{i i} o_{j i}(A) A^{k}, y_{i}\right) \\
& \left(x_{i} A^{k} B, x_{j}\right)=\left(y_{j} \psi_{i j}(A) A^{k}, x_{j}\right)=-\left(x_{j} \psi_{i j}(A) A^{k}, y_{j}\right) \\
& \left(x_{i} A^{k}, x_{j} B\right)=\left(x_{i} A^{k}, y_{i} \psi_{j i}(A)\right)=\left(x_{i} \psi_{j i}(A) A^{k}, y_{i}\right) \\
& \left(y_{i} A^{k} B, y_{j}\right)=\left(x_{j} \eta_{i j}(A) A^{k}, y_{j}\right) \\
& \left(y_{i} A^{k}, y_{j} B\right)=\left(y_{i} A^{k}, x_{i} \eta_{j i}(A)\right)=-\left(x_{i} \eta_{j i}(A) A^{k}, y_{i}\right) .
\end{aligned}
$$

Thus $B$ is self-adjoint if and only if

$$
\begin{aligned}
& \left(x_{j} \varphi_{i j}(A) A^{k}, y_{j}\right)=\left(x_{i} \rho_{j i}(A) A^{k}, y_{i}\right) \\
& \left(x_{j} \psi_{i j}(A) A^{k}, y_{j}\right)=-\left(x_{i} \psi_{j i}(A) A^{k}, y_{i}\right) . \\
& \left(x_{j} \eta_{i j}(A) A^{k}, y_{j}\right)=-\left(x_{i} \eta_{j i}(A) A^{k}, y_{i}\right) .
\end{aligned}
$$

Since $\left(x_{i} A^{k}, y_{i}\right)=1$ if $k=d_{i}-1,=0$ otherwise, we have, exactly as in the symmetric case,

$$
\varphi_{i j}=\delta_{i}^{-1} \rho_{j i} \delta_{j}, \psi_{i j}=-\delta_{i}^{-1} \psi_{j i} \delta_{j}, \eta_{i j}=-\delta_{i}^{-1} \eta_{j i} \delta_{j} .
$$

To $B$ we now assign the $2 r \times 2 r$ matrix $\left(\beta_{u, v}(\lambda)\right)$ where

$$
\beta_{2 i-1,2 j-1}=\varphi_{i, j}
$$




$$
\begin{aligned}
& \beta_{2 i-1,2 j}=\phi_{i, j} \\
& \beta_{2 i, 2 j-1}=\eta_{i, j} \\
& \beta_{2 i, 2 j}=\rho_{i, j}
\end{aligned}
$$

i.e. we arrange the elements $\varphi, \psi, \eta, \rho$ in $2 \times 2$ blocks

$$
\left(\begin{array}{ll}
\varphi_{i j} & \psi_{i j} \\
\eta_{i j} & \rho_{i j}
\end{array}\right)
$$

If now $(\delta)$ is the matrix with the blocks

$$
\left(\begin{array}{cc}
0 & \delta_{i} \\
-\delta_{i} & 0
\end{array}\right)
$$

on the main diagonal and zeros elsewhere, condition (2) in matrix form is $(\delta)^{-1}(\beta)^{\prime}(\delta)=(\beta)$.

If $B$ corresponds to $(\beta)$, clearly $B=0$ if and only if $\varphi_{i j}, \psi_{i j}, \eta_{i j}, \rho_{i j}$ are all $\equiv 0\left(\bmod \delta_{j}\right)$, i.e. $(\beta)=(\alpha)(\delta)$ for some $(\alpha)$ in $(F[\lambda])_{-r}$. It is now clear that the map $B$ into $\beta$ gives an isomorphism of $(\mathfrak{s}((\mathrm{A}))$ and $\Re / \Re$.

(c) This case is treated in [6], Chapt. 3, where however the defining relation for $\Omega$ is

$$
\delta_{i} \beta_{i j} \equiv 0\left(\bmod \delta_{j}\right) .
$$

However, the condition $\delta_{i} \beta_{i j} \equiv 0\left(\bmod \delta_{j}\right)$ is clearly equivalent to $(\delta)^{-1}(\beta)^{\prime}(\delta)$ being in $(F[\lambda])_{r}$, i.e. to the existence of $(\gamma)$ in $(F[\lambda])_{r}$ such that $(\beta)^{\prime}(\delta)=$ $(\delta)(\gamma)$

(d) Let $\Im$ of be type $D$, then $\Im=F \cdot 1 \oplus V, 1$ the identity element and $V$ a vector space of dimension at least two and with non-degenerate symmetric scalar product $(x, y)$. $\Im$ can be considered as a subspace of the Clifford algebra $C$ determined by $V$, and the product in $\Im$ can be written as $x \circ y=x y+y x$, where $x y$ is the product in $C$. The vector space $C$ can be identified with the vector space of the exterior algebra $E$ over $V$, with multiplication $x \wedge y$, in such a way that $x y=x \wedge y+(x, y) 1$ for $x, y$ in $V$ (See [3]). Let now $a=\alpha 1+v, \alpha \in F, v \in V$ be an element of $\mathfrak{\Im}$. Clearly $\mathfrak{E}_{\mathfrak{S}}((a))=\mathfrak{\Im} \mathfrak{s}((v))$, and $\mathfrak{c} \mathfrak{\Im}((a))=\mathfrak{\Im}$ if $v=0$. Let now $v \neq 0$ : we have to show $\mathbb{E}_{\mathfrak{s}}((v))=(v)$. Let $w \in V$ and $w \in \mathbb{E} \mathfrak{s}((v))$. As shown in $\S 1, v w=w v$, but $v w=v \wedge w+(v, w) 1$, so $v \wedge w=w \wedge v=0$, thus $w$ is in $(v)$. This proves $\varsigma_{\Im}((v))=(v)$ if $v$ is not zero, and completes the proof of the theorem.

CoRollaRy 2.1. If $\mathfrak{\Im}$ is a central simple Jordan algebra of type $B$ with enveloping associative algebra $\mathfrak{A}$ and $x$ is any e'ement of $\mathfrak{\Im}$, then the enveloping associative algebra of $\mathfrak{S}_{\mathfrak{s}}((x))$ is $\mathfrak{V}_{\mathfrak{g}}((x))$. The same statement does not hold if $\mathfrak{\Im}$ is of type $C$. 
Proof. As usual, we assume the base field $F$ is algebraically closed, and consider first the case of a nilpotent element $x$. Then, in the notation of previous theorem, $[\mathfrak{r}((x))$ can be identified with the associative algebra $\Re / \Re, \Re$ the subalgebra of $(F[\lambda])$. of matrices $(\alpha)$ such that $(\delta)^{-1}(\alpha)^{\prime}(\delta)$ is in $(F[\lambda])$, and $\Re$ the ideal of matrices of the form $(\alpha)(\delta)$, while $\mathfrak{C}_{\Im}((x))$ is the Jordan subalgebra $(H+\mathfrak{R}) / \mathfrak{R}$ of $\Re / \Re, H$ denoting the matrices $(\alpha)$ such that $(\delta)^{-1}(\alpha)^{\prime}(\delta)=(\alpha)$. It is sufficient to prove that the enveloping algebra of $H$ in $(F[\lambda])$. contains every element of $\Re$. If $r>1, H$ contains elements $\varphi e_{i i}$ and $\varphi e_{i j}+\delta_{i} \delta_{j}^{-1} \varphi e_{j i}$ for $1 \leqq i<j \leqq r$ and $\varphi$ any polynomial. Thus the enveloping algebra contains $e_{i i}\left(\varphi e_{i j}+\right.$ $\left.\delta_{i} \delta_{j}^{-1} \varphi e_{j i}\right)=\varphi e_{i j}$ for $i<j$ and also $\delta_{i} \delta_{j}^{-1} \varphi e_{j i}=\left(\varphi e_{i j}+\delta_{i} \delta_{j}^{-1} \varphi e_{j i}\right) e_{i i}$ as well as $\varphi e_{i i}$ for all $i$; however the elements $\varphi e_{i j}, i \leqq j$, and $\psi e_{i j}$ with $\psi \equiv 0$ $\left(\bmod \delta_{i} \delta_{j}^{-1}\right)$ for $i>j$, clearly are a basis for $\Re$. Note that if $r=1$, then $\mathfrak{S}_{\Im}((x))=\mathcal{C}_{2}((x))$. If now $x$ is not nilpotent, we write $x=\sum \lambda_{i} e_{i}+n_{i}$, $e_{i}$ idempotent and $n_{i}$ nilpotent as before, and set $\mathfrak{N}_{i}=e_{i} \mathfrak{Y}\left(e_{i}, \mathfrak{\Im}_{i}=e_{i} \mathfrak{\Im} e_{i}\right.$. Then $\quad\left(\mathfrak{S}_{\mathfrak{A}}((x))=\sum_{i} \oplus \mathfrak{S}_{\mathfrak{H}}\left(\left(n_{i}\right)\right), \quad\left(\mathfrak{S}_{\mathfrak{s}}((x))=\sum \oplus \mathfrak{S}_{\mathfrak{\Im}_{i}}\left(\left(n_{i}\right)\right)\right.\right.$, therefore the enveloping algebra of $(5 \Im((x))$ is $(\widetilde{r}((x))$. If $\Im$ is of type $C$, and $A$ is a nilpotent $2 n \times 2 n$ symplectic-symmetric element of $\mathfrak{\Im}$ of index of nilpotency $n$, i.e. $r=1, \delta_{1}=\lambda^{n}$, then it is easy to see that $\widetilde{c}_{\Im}((x))=(x)$ and is a commutative associative algebra, whereas $\mathcal{E}_{\mathfrak{A}}((x))$ is not commutative.

TheOREM 2.2. Let $\Im$ be a central simple Jordan algebra, $n$ its degree and $n+(1 / 2) n(n-1) s$ its dimension [thus $s=1,2,4,8$ if $n \geqq 3, s=1$, $2,4$ if $n>3, s \geqq 1$ if $n=2]$. Let $x$ be an element of $\mathfrak{s}$ with invariant factor degrees $d_{1} \geqq d_{2} \geqq \cdots \geqq d_{r}$, as defined before. Then $\mathbb{S}_{\mathfrak{s}}((x))$ has dimension $\sum_{k=0}^{r-1}(s k+1) d_{k+1}$. If $s=8$, we assume characteristic $\neq 2$ or 3.

Proof. We may assume the base field is algebraically closed.

(a) Let $\Im$ be of type $A$ : this is the theorem of Frobenius and follows from Th. 2.1, part C). Here $s=2$.

(b) $\mathfrak{S}$ of type $B$. First let $x$ be nilpotent. We merely have to calculate the dimension of the space of matrices $(\beta)$ with $(\delta)^{-1}(\beta)^{\prime}(\delta)=(\beta)$ and subtract the dimension of the $(\beta)$ of the form $(\alpha)(\delta)$, i.e. reduce $\beta_{i j} \bmod \delta_{j}$. Clearly $(\beta)$ is determined by the elements $\beta_{i j}$ with $i \leqq j$ and $\beta_{i j}$ of degree $\leqq d_{j}$, thus the dimension is $d_{1}+2 d_{2}+\cdots+r d_{r}$, and here $s=1$. If $x$ is not nilpotent, $x=\sum \alpha_{i} \varepsilon_{i}+n_{i}$, and $\mathfrak{c}_{\Im}((x))$ is a direct sum of the algebras $\mathfrak{S}_{\mathscr{R}}((n)), \quad \Re=\mathfrak{\Im}_{1}\left(e_{i}\right)$ and $n=n_{i}$. The $\mathfrak{\Im}_{1}\left(e_{i}\right)$ are again algebras of all symmetric matrices (of degree $\geqq 1$ ), and the invariant factor degree $d_{k}$ of $x$ is the sum of the $d_{k}\left(n_{i}\right)$. Thus the formula holds also for $x$.

(c) Type $C$. Again we need only consider nilpotent elements, and 
the calculation of the dimension is like that for symmetric matrices, using Th. $2.1 \mathrm{~b}$ and its proof.

(d) $\Im$ of type $D$. Let $x=\alpha 1+v, v \in V$, using the notation of the proof of Cor. 2.1. If the minimum polynomial $\delta_{1}$ has degree one, then $v=0, d_{1}=d_{2}=1$, and $\mathfrak{\zeta}_{\mathfrak{s}}((x))=\mathfrak{\Im}$. Here $2+s=$ dimension of $\Im$, so $\sum_{k=0}^{r-1}(s k+1) d_{k+1}=d_{1}+(s+1) d_{2}=s+2$. Let now the minimum polynomial have degree two, then $d_{1}=2, d_{2}=0$, and $v$ is not zero, so $\mathfrak{c}_{\Im}((x))$ $=(x)$ has dimension $2=\sum_{k=0}^{r-1}(s k+1) d_{k+1}$.

(e) $\Im$ the exceptional algebra, type $E$. We use the previously mentioned result of Professor Jacobson that if $x$ is a nilpotent element of $\Im$, with $x^{n}=0, x^{n-1} \neq 0$, then $(x)$ can be imbedded in a direct sum of algebras $H^{(i)}$ of all $n_{i} \times n_{i}$ symmetric matrices with $n=n_{1} \geqq n_{2} \geqq$ $\cdots \geqq n_{r}$. The identity element of $\mathfrak{\Im}$ is in $(x)$ and $\mathfrak{\Im}$ has degree 3 , so $r$ is at most 3 , and $x$ satisfies a cubic polynomial over the center $F$. Thus we have only a small number of cases to consider. Let the cubic polynomial be $\left(\lambda-\alpha_{1}\right)\left(\lambda-\alpha_{2}\right)\left(\lambda-\alpha_{3}\right), \alpha_{i} \in F$.

(1) The $\alpha_{i}$ are all equal, say to $\alpha_{1}$. Since $\mathbb{F}_{\Im}\left(\left(x-\alpha_{1} 1\right)\right)=\mathbb{} \mathfrak{s}((x))$, we may replace $x$ by $x-\alpha_{1} 1$, and thus have $x$ nilpotent.

(i) Let $x^{3}=0, x^{2} \neq 0$. Since no element of a $2 \times 2$ or $1 \times 1$ matrix algebra can satisfy this condition, $H^{(1)}$ must be of degree 3 , and $H^{(i)}=0$ for $i>1$. Then $H^{(1)}$ contains primitive orthogonal idempotents $e_{i}$ with sum 1 , and elements $u_{12}, u_{13}, u_{23}$ with $u_{i j} \circ u_{i j}=4\left(e_{i}+e_{j}\right), u_{i j} \circ u_{j k}$ $=u_{i k}$ for $i, j, k$ distinct, and $e_{i} \circ u_{i j}=(1 / 2) u_{i j}$. By Theorem 9.1 of [9], $\Im$ can be represented as $H\left(C_{3}\right)$ with these elements as matrix units and involution $\sum c_{i j} e_{i j} \rightarrow \sum \bar{c}_{i j} e_{j i},\left(e_{i i}=2 e_{i}, e_{i j}+e_{j i}=u_{i j}\right)$. The element $x$ is represented by a matrix $N$ with elements in $F$. Since $N^{3}=0, N^{2} \neq 0$, and $N$ is a $3 \times 3$ symmetric matrix, it follows from the remarks after Lemma 2.1 that there is an orthogonal matrix $T$ with elements in $F$ such that $T N T^{-1}=M$,

$$
M=\left(\begin{array}{ccc}
0 & 0 & 1 \\
0 & 0 & \alpha \\
1 & \alpha & 0
\end{array}\right) \quad \alpha \in F \text { and } \alpha^{2}=-1
$$

since also $M^{3}=0, M^{2} \neq 0$. The matrix $T$ is in the nucleus of $C_{3}$ (i.e. associates with all elements), and so $X \rightarrow T X T^{-1}=X^{A}$ is an automorphism $A$ of $H\left(C_{3}\right)$. Using the matrix units $e_{i}^{A}, u_{i j}^{A}$, by Th. 9.1 of [9] we may represent $\Im$ as $H\left(C_{3}\right)$ with involution the ordinary conjugate transpose operation with $x$ being represented by $M$. Since $M$ has coefficients in the center of $C$, by Proposition $1.3 \mathrm{~b}, \mathfrak{c}_{\Im}((M))$ is just the set of matrices commuting with $M$ : these turn out to be all matrices 
of the form

$$
X=\gamma_{1} e_{11}+\gamma_{2} e_{22}+\gamma_{3} e_{33}+x_{3} e_{12}+\bar{x}_{3} e_{21}+y_{2} e_{13}+\bar{y}_{2} e_{31}+z_{1} e_{23}+\bar{z}_{1} e_{31}
$$

such that the $\gamma_{i}$ are in $F, \bar{x}_{3}=x_{3}, \bar{y}_{2}=y_{2}, \bar{z}_{1}=z_{1}=\alpha y_{2}$

and

$$
\gamma_{1}+\alpha x_{3}=\gamma_{3}=\gamma_{2}-\alpha x_{3} .
$$

Thus the $x_{i}, y_{i}, z_{i}$ are in $F$, and $X=\left(\gamma_{1}+\alpha x_{3}\right) 1+\alpha z_{1} M+y_{2} M^{2}$. Hence $\mathfrak{c}_{\Im}((x))=(x)$. Also, $d_{1}=3, d_{2}=d_{1}=0$ and $\sum(8 k+1) d_{k+1}=3=$ dimension of $(x)$.

(ii) $x^{2}=0$ : then $H^{(1)}$ is of degree two, so $H^{(2)}$ must be of degree one and equal to $\mathrm{Fe}, e$ a primitive idempotent. We note for future reference that $H^{(1)} \oplus H^{(2)}$ is contained in $\mathfrak{\Im}_{e}(1)+\mathfrak{\Im}_{e}(0)=\{a$ in $\Im$ such that $e \circ a=a$ or $e \circ a=0\}$ and so $e$ is in $\complement_{5}((x))$. $H^{(1)}$ contains primitive orthogonal idempotents $e_{1}, e_{2}$ and an element $u_{12}$ with $u_{12} \circ u_{12}=4\left(e_{1}+e_{2}\right)$. Write $e_{3}$ for $e$, then there are elements $v_{13}, w_{23}$ in $\Im$ such that $v_{13} \circ v_{13}=$ $4\left(e_{1}+e_{3}\right), w_{23} \circ w_{23}=4\left(e_{2}+e_{3}\right), u_{12} \circ v_{13}=w_{23}, u_{12} \circ w_{23}=v_{13}$, and $v_{13} \circ w_{23}=$ $u_{12}$ (this can be seen, for instance, by writing $\Im$ as $H\left(C_{3}\right)$ with $2 e_{i}=e_{i i}$, so that $u_{12}=c e_{12}+\bar{c} e_{21}$ where $c \bar{c}=1$ in $C$, and setting $v_{13}=c e_{13}+\bar{c} e_{31}$, $\left.w_{23}=e_{23}+e_{32}\right)$. As before we can write $\mathfrak{\Im}$ as $H\left(C_{3}\right)$ using the $o_{i}, u_{12}, v_{13}$, $w_{23}$ as matrix units : then $x$ is a linear combination of $e_{1}, e_{2}, u_{12}$, and $e_{3}$ and so is represented by a matrix with elements in $F$. Since $x^{2}=0$, the coefficient of $e_{3}$ must be zero, and as before the $2 \times 2$ symmetric matrix $\gamma_{1} e_{1}+\gamma_{2} e_{2}+\gamma_{3}\left(e_{12}+\epsilon_{21}\right), \gamma_{i}$ in $F$, may be transformed to the form $\alpha\left(e_{1}-e_{2}\right)+e_{12}+e_{21}$ by a $2 \times 2$ orthogonal matrix $T$ with coefficients in

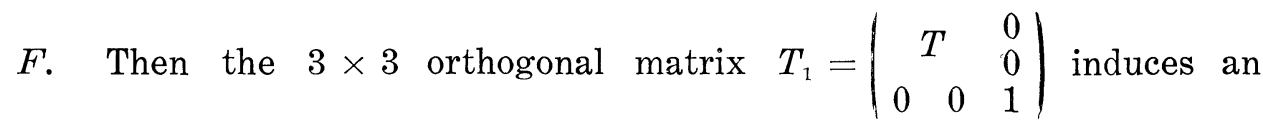
automorphism of $H\left(C_{3}\right)$, taking $x$ into

$$
N=\left(\begin{array}{ccc}
\alpha & 1 & 0 \\
1 & -\alpha & 0 \\
0 & 0 & 0
\end{array}\right) \quad \alpha^{2}=-1 \text {. }
$$

Again we have only to find the matrices commuting with $N$, which turn out to have the form

$$
\left(\begin{array}{ccc}
\beta_{1} & (1 / 2) \alpha\left(\beta_{2}-\beta_{1}\right) & \alpha z_{3} \\
(1 / 2) \alpha\left(\beta_{2}-\beta_{1}\right) & \beta_{2} & z_{3} \\
\alpha \bar{z}_{3} & \bar{z}_{3} & \beta_{3}
\end{array}\right), \quad \beta_{i} \text { in } F, z_{3} \text { in } C .
$$

Thus the dimension of $\mathfrak{c}_{\Im}((x))$ is $3 \cdot \operatorname{dim} F+\operatorname{dim} C=11$; also $d_{1}=2$, $d_{2}=1, d_{3}=0$ so $\sum(8 k+1) d_{k+1}=2+9=11$.

(2) The cubic polynomial of $x$ has two distinct roots. Then $(x)$ contains two orthogonal idempotents $e, f$ with sum 1 , which we may write as $e=e_{3}, f=e_{1}+e_{2}$, where the $e_{i}$ are orthogonal primitive 
idempotents in $\mathfrak{F}$. Then $x=\gamma_{1} f+n+\gamma_{3} e$, where $n$ is nilpotent, in fact $n^{2}=0, e \circ n=0, f \circ n=n$, and $e, f, n$ are all in $(x)$. Note that $(x) \subseteq$ $J_{e}(0)+J_{e}(1)$ and $e$ is in $\mathfrak{F}_{\Im}((x))$. Suppose first that $n=0$. Then $\mathfrak{F}_{\Im}((x))$ $=\mathfrak{c}_{\Im}(e)=\mathfrak{c}_{\Im}(f)=\Im_{e}(0)+J_{e}(1)$ and has dimension 11 , while $d_{1}=2, d_{2}=1$ and $\sum(8 k+1) d_{k+1}=11$. Now suppose $n \neq 0$, and let $y$ be in $\varsigma_{\Im}((x))$. Since $y$ o-commutes with $e, y=y_{0}+y_{1}, y_{i}$ in $\mathfrak{\Im}_{e}(i)$. Since $\mathfrak{\Im}_{e}(0)$ is an algebra, $y_{0}$ o-commutes with $n$ in $\mathfrak{\Im}_{e}(0)$-a simple algebra of degree two; thus by part (d) of this theorem, $y_{0}$ is a linear combination of $f$ and $n$ since $n$ is not in the center of $\Im_{e}(0)$. Also, $y_{1}$ is a scalar multiple of $e$ since $\mathfrak{\Im}_{e}(1)=F e$; thus $y=y_{0}+y_{1}$ belongs to $(x)$ and $\widetilde{c}_{\mathfrak{s}}((x))=(x)$ and has dimension 3. $d_{1}=3, d_{2}=0$ and $\sum(8 k+1) d_{k+1}=3$.

(3) The cubic polynomial has 3 distinct roots. Then $(x)=F e_{1}+$ $\mathrm{Fe}_{2}+\mathrm{Fe}_{3}, e_{i}$ mutually orthogonal, and $\mathfrak{c}_{\mathfrak{s}}((x))$, being the intersection of the $\Im_{e}(0)+\Im_{e}(1)$ for $e=e_{1}, e_{2}, e_{3}$ respectively, equals $(x)$. Here $d_{3}=3$, $d_{2}=d_{1}=0$, and $\sum(8 k+1) d_{k+1}=3=\operatorname{dim} \varsigma_{\Im}((x))$. Incidentally, we also note that for each $x$ in $\mathfrak{\Im}$ there is a representation of $\mathfrak{\Im}$ as $H\left(C_{3}\right)$ such that the matrices of $\mathfrak{c} \Im((x))$ are just those commuting with the matrices of $(x)$.

COROLlary 2.2. Let $\mathfrak{\Im}$ be a central simple Jordan algebra of degree $n,(n \geqq 1), x$ an element of $\mathfrak{\Im}$. Then the minimum polynomial of $x$ in $\Im$ has degree at most $n$, and equals $n$ if and only if $\mathfrak{s}((x))=(x)$. Elements $x$ with minimum polynomial of degree $n$ always exist.

Note. such elements with minimum polynomial of degree equal to the degree of the algebra are a natural generalization of non-derogatory $n \times n$ matrices in the Jordan algebra $\left(F_{n}\right)_{j}$.

Proof of the Corollary. Since the dimension of $(x)$ is $d_{1}$, the degree of the minimum polynomial of $x$, and $(x)$ is always contained in ${ }_{5} \Im((x))$, we see that $\sum_{k=0}^{r-1}(s k+1) d_{k+1}=d_{1}$ if and only if $d_{2}=\cdots=d_{r}=0$. But since $d_{1}+\cdots+d_{r}=n$, this means $d_{1}=n$. If the base field is algebraically closed, hence infinite, and $e_{1}, \cdots, e_{n}$ are primitive orthogonal idempotents with sum 1 , then $x=\alpha_{1} e_{1}+\cdots+\alpha_{n} e_{n}$ is of degree $n$ if the $\alpha_{i}$ are distinct. Since the degree of the minimum polynomial is unaffected by field extension, there must exist elements with $d_{1}=n$ in a central simple $\Im$ over any base field.

We next consider the double centralizer $\mathfrak{E}_{\Im}(\sqrt{5} \Im(x))$, and prove that it is always equal to $(x)$ : a known theorem for $\mathfrak{S}$ the algebra of all $n \times n$ matrices over a field. We can also prove this for $\Im$ a Jordan algebra analogous to a finite dimensional Jordan algebra of type $A, B$, or $C$ but obtained from a simple ring with minimum condition instead of a simple algebra. 
THEOREM 2.3. Let $\mathfrak{A}$ be an involutorial simple ring with minimum condition, $F$ the set of self-adjoint elements of the center of $\mathfrak{A}$, and $\mathfrak{\Im}$ the set of self-adjoint elements of $\mathfrak{A}$, regarded as a Jordan algebra over $F$. Let $x$ be an algebraic element of $\mathfrak{\Im}$, i.e. $(x)$ is finite dimensional over F. Then $\mathfrak{E}_{\Im}\left(\mathfrak{E}_{\Im}((x))\right)=(x)$.

Proof. We can also consider $\mathfrak{A}$ as an algebra over $F$, and if $K$ is a finite extension field of $F$ containing the eigenvalues of $x$, form $\mathfrak{A} \bigotimes_{F} K, \mathfrak{I} \bigotimes_{F} K$, noting that the involution in $\mathfrak{A}$ can be extended to $\mathfrak{A} \otimes K$ by letting it be the identity on $K=1 \otimes K$ and that $\mathfrak{\Im} \otimes K$ is then the set of self-adjoint elements of $\mathfrak{A} \otimes K$. We then need only prove that if $x$ is an algebraic element of $\mathfrak{\Im} \otimes K$ with eigenvalues in $K$, then $(x)$ is its own double centralizer.

Case 1. Let $E$, the center of $\mathfrak{A}$, equal $F$. Then $\mathfrak{A} \otimes K$ is again an involutorial simple ring with minimum condition so that we may as well assume $\dot{F}$ already contained the eigenvalues of $x$. We may represent $\mathfrak{A}$ as the algebra (over $F$ ) of all linear transformations on a finite dimensional vector space $V$, over an involutorial division ring $D$ with center $F$, with a non-degenerate scalar product which either is hermitian or else is symplectic and $D=F$.

(a) Hermitian scalar product. We use the following lemma:

LEMMA 2.3. Let $V$ be a vector space of dimension $n \geqq 1$ with nondegenerate hermitian scalar product over a division ring $D$. Let $F$ be the subfield of. self-adjoint elements of $D, \mathfrak{\Im}$ the Jordan algebra, over $F$, of self-adjoint linear transformations in $D$. Let $A$ be an element of $\mathfrak{\Im}$ such that $A^{n}=0, A^{n-1} \neq 0$ (set $\left.A^{0}=1\right)$. Then $\mathfrak{E}_{\mathfrak{s}}\left(\mathfrak{S}_{\Im}((A))\right)=(A)$.

Proof. First consider the case $n=1$, that is $V=D$ (as left $D$

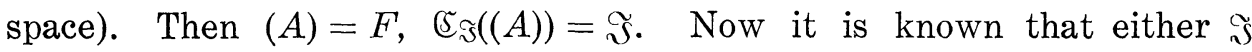
generates $D$ or else $\mathfrak{\Im}=F$ ([7], p. 187). In either case, $\mathfrak{c}_{\Im}(\Im)=$ $\left.\mathfrak{S}_{\Im}(\mathfrak{\Im} \Im(A))\right)=(A)=$ center of $\mathfrak{\Im}=F$. Let now $n>1$. By Lemma 2.1 we can find a basis for $V$ of the form $\left(v, v A, \cdots, v A^{n-1}\right)$ such that $\left(v A^{i}, v A^{j}\right)=\lambda$, a self-adjoint non-zero element of $D$, if $i+j=n-1$, and $\left(v A^{i}, v A^{j}\right)=0$ otherwise. Let $B$ be a self-adjoint linear transformation commuting with $\mathfrak{A}$, and let $v B=\beta_{0} v+\beta_{1} v A+\cdots+\beta_{n-1} v A^{n-1}, \beta_{i}$ in $D$. Then

$$
\begin{aligned}
& \left(v B, v A^{n-1-i}\right)=\beta_{i}\left(v A^{i}, v A^{n-1-i}\right)=\beta_{i} \lambda \\
& \left(v, v A^{n-1-i} B\right)=\left(v, \beta_{i} v A^{n-1}\right)=\lambda \beta_{i}^{\prime}
\end{aligned}
$$

for all $i$, and conversely, any linear transformation $B$ commuting with $A$ such that the $\beta_{i}$ satisfy $\beta_{i} \lambda=\lambda \beta_{i}^{\prime}$ is self-adjoint, $(i=0,1, \cdots, n-1)$. For $\gamma$ in $D$, let $\gamma^{*}=\lambda \gamma^{\prime} \lambda^{-1}$. Then $\gamma \rightarrow \gamma^{*}$ is an involution in $D$, and $\beta_{i}^{*}$ 
$=\beta_{i}$. The self-adjoint elements of this new involution either generate $D$ or else lie in the center of $D$. First suppose they lie in the center of $D$ : then $\beta_{i}^{*}=\beta_{i}^{\prime}=\beta_{i}$ so the $\beta_{i}$ are in $F$, and $B$ is in $(A),(B=$

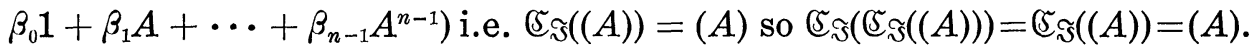
Next suppose that the self-adjoint elements of $*$ generate $D$, and let $M$ be in $\mathfrak{\Im}_{\Im}\left(\Im_{\Im}((A))\right)$. Since $A$ is in $\Im_{\Im}((A)), M$ commutes with $A$ and so $v M$ $=\mu_{0} v+\cdots+\mu_{n-1} v A^{n-1}$. Also $M$ commutes with the linear transformation $B_{0}(\beta): \rho v A^{i} \rightarrow \rho \beta v A^{i}(i=1, n-1$, in $D)$ for every $\beta$ such that $\beta=\beta^{*}$. Thus $v B_{0} M=\beta \mu_{0} v+\cdots+\beta \mu_{n-1} v A^{n-1}=v M B_{0}=\mu_{0} \beta v+\cdots+\mu_{n-1} \beta v A^{n-1}$ so that the $\mu_{i}$ commute with all such $\beta$, and since the latter generate $D$, every $\mu_{i}$ is in the center of $D$, so $\mu_{i}^{*}=\mu_{i}^{\prime}=\mu_{i}$ and the $\mu_{i}$ are in $F$. Thus $B$ is in $(A)$. This completes the proof of the lemma.

Let now $A$ be an algebraic element of $\Im$ with eigenvalues in $F$ : thus $A=\sum \lambda_{i} E_{i}+N_{i}$ with $\lambda_{i}$ distinct, in $F$, and $N_{i}$ nilpotent, $E_{i}, N_{i}$ being in $(A)$. First let the degree of the minimum polynomial of $A$ on $V$ equal the dimension of $V$. Then $V$ is a direct sum of the non-isotropic mutually orthogonal subspaces $V_{i}=V E_{i}$ and the index of nilpotency of $N_{i}=E_{i} N_{i}$ equals the dimension of $V_{i}$. Since the $E_{i}$ are in $(A)$ and so in $\mathfrak{\Im}_{\Im}((A))$, every element of $\mathfrak{\Im}_{\Im}\left(\mathfrak{C}_{\Im}((A))\right)$ maps each $V_{i}$ into itself, commutes with $N_{i}$ on $V_{i}$, and is self-adjoint on $V_{i}$. By the lemma the induced transformation on $V_{i}$ is a polynomial $\varphi_{i}(A)$ with coefficients in $F$. Since the minimum polynomials of $A$ in $V_{i}$ are relatively prime in pairs, there is a polynomial $\varphi(\lambda)$ such that $\varphi(A) E_{i}=\varphi_{i}(A) E_{i}$, that is $\varphi(A)$ induces $\varphi_{i}(A)$ on $V_{i}$. Thus every element of $\mathbb{E}_{\Im}\left(\mathbb{S}_{\Im}((A))\right)$ is a polynomial in $A$. Finally, consider the general case. Then $V$ is a direct sum of cyclic mutually orthogonal subspaces $W_{i}$ such that $W_{i+1}$ is a homomorphic image of $W_{i}$ as $A$-space and the minimum polynomial of $A$ on $W_{i}$ has degree equal to the dimension of $W_{i}$. Since the orthogonal projections of $V$ on $W_{i}$ are self-adjoint and in $\mathfrak{c}_{\Im}((A))$, every element $C$ of $\mathfrak{\Xi}_{\Im}\left(\mathbb{S}_{\Im}((A))\right)$ maps each $W_{i}$ on itself. Let $S_{i}$ be a self-adjoint linear transformation of $W_{i}$ into itself which commutes with $A$ on $W_{i}$. $S_{i}$ can be extended to $V$ by letting it act as zero on the $W_{j}$ with $j \neq i$, and will then belong to $\Xi_{\Im}((A))$. Thus $C$ commutes with $S_{i}$, and so on each $W_{i}, C$ is equal to a polynomial $\psi_{i}(A)$. Finally, we must show that all the $\psi_{i}(A)$ may be assumed equal. Let $x_{1}$ be a cyclic generator of $W_{1}$, and $x_{2}$ its image under an $A$-homomorphism of $W_{1}$ onto $W_{2}$. Then $x_{2}$ is a cyclic generator of $W_{2}$. Denote by $T$ the mapping of $W_{1}$ on $W_{2}$ with $x_{1} T=x_{2}$, and define $T=0$ on $W_{i}$ for $i \geqq 2$. Let $T^{\prime}$ be the adjoint of $T$. Since $\left(W_{j} T, W_{1}\right)=0,\left(W_{j}, W_{1} T^{\prime}\right)=0$ for all $j$, so $W_{1} T^{\prime}=0$. Let $S=T+T^{\prime}$, then $S=T$ on $W_{1}, S$ is self-adjoint and since $T A=A T$, also $T^{\prime} A=A T^{\prime}$ and $S A=A S$. Thus $S$ is in $\complement_{\Im}((A)), S C=C S$, and $x_{1} S=x_{2}$. For all $i$, $x_{i} C=x_{i} \psi_{i}(A)$. Since $x_{2}=x_{1} S, x_{2} \psi_{2}(A)=x_{2} C=x_{1} S C=x_{1} C S=x_{1} \psi_{1}(A) S=$ $x_{1} S \psi_{1}(A)=x_{2} \psi_{1}(A)$. Thus $\psi_{1}(A)=\psi_{2}(A)$ on $W_{2}$, and in the same way 
$\psi_{1}(A)=\psi_{i}(A)$ on $W_{i}$ for all $i$. Thus $C=\psi_{1}(A)$ on $V$.

(b) Skew-symmetric scalar product: We now write $A$ for the element $x$ of $\Im$. By Lemma 2.2, we can write $V$ as a direct sum of cyclic totally isotropic spaces $W_{i}, W_{i}^{\prime}$ such that $W_{i}+W_{i}^{\prime}$ is non-isotropic and orthogonal to $W_{j}+W_{j}^{\prime}$ for $i \neq j, W_{i}$ is isomorphic to $W_{i}^{\prime}$, and $W_{i+1}$ is a homomorphic image of $W_{i}$ as $(A)$ modules. Let $V_{i}=W_{i}+W_{i}^{\prime}$ and $A_{i}$ the restriction of $A$ to $V_{i}$. Let $\widetilde{\Im}_{i}$ be the algebra of self-adjoint linear transformations on $V_{i}$; by Corollary $2.2, \quad \varsigma_{\Im_{i}}\left(\left(A_{i}\right)\right)=\left(A_{i}\right)$, since the degree of $\widetilde{\Im}_{i}$ equals the dimension of $W_{i}$. Let $E_{i}$ be the self-adjoint projection on $V_{i}$, and let $C$ be in $\widetilde{c}_{\Im}\left(\widetilde{S}_{\Im}((A))\right)$; then $C$ commutes with the $E_{i}$ and maps $V_{i}$ on itself, inducing $C_{i}$ on $V_{i}$. Since $C_{i}$ is in $\widetilde{S}_{\Im_{i}}\left(\left(A_{i}\right)\right)$, $C_{i}$ is a polynomial $\varphi_{i}(A)$. To show the $\varphi_{i}$ are all equal, we choose cyclic generators $x_{i}, x_{i}^{\prime}$ of $W_{i}, W_{i}^{\prime}$ and define a linear transformation $T$ by: $T A=A T, x_{i} T=x_{i+1}, x_{i}^{\prime} T=x_{i+1}^{\prime}$, and $T$ is zero on $V_{j}$ for $j \neq i$. As in the symmetric case, the adjoint $T^{\prime}$ of $T$ is zero on $V_{i}$, so if we set $S=$ $T+T^{\prime}$, then $x_{i} S=x_{i+1}, x_{i}^{\prime} S=x_{i+1}^{\prime}$ and $S$ is in $\left(\widetilde{s}((A))\right.$. Then $x_{i} S C=$ $x_{i+1} C=x_{i+1} \varphi_{i+1}(A)=x_{i} C S=x_{i+1} \varphi_{i}(A)$, and similarly, $x_{i+1}^{\prime} \varphi_{i+1}(A)=x_{i+1}^{\prime} \varphi_{i}(A)$. Thus $C=\varphi(A)=\varphi_{1}(A)$ on all of $V$. This completes the proof of Case 1 .

Case 2. $E$, the center of $\mathfrak{A}$, is a quadratic extension of $F$. Just as in the case of finite dimensional algebras, the ring $\mathfrak{U} \bigotimes_{F} E$ is a direct sum of two copies $\mathfrak{A}_{1}, \mathfrak{U}_{2}$ of $\mathfrak{A}$ and if we extend the involution of $\mathfrak{A}$ to $\mathfrak{A} \otimes E$ by letting it be the identity on $1 \otimes E$ (i.e. if $a \rightarrow a^{\prime}$ is the involution in $\mathfrak{A}$, we set $(a \otimes e)^{\prime}=a^{\prime} \otimes e$ for $e$ in $\left.E\right)$ then the involution

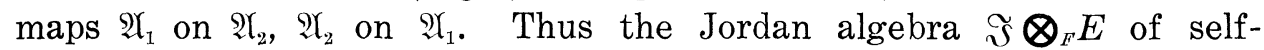
adjoint elements of $\mathfrak{U} \bigotimes_{F} E$ is isomorphic to the set of elements $a_{1} \oplus a_{1}^{\prime}$ of $\mathfrak{A}_{1} \oplus \mathfrak{A}_{2}$, where $a_{1}$ is in $\mathfrak{A}_{1}$ and $a_{1}^{\prime}$ the image of $a_{1}$ under the involution; as $\mathfrak{H}_{1}$ is isomorphic to $\mathfrak{A}, \mathfrak{\Im} \bigotimes_{F} E$ is also isomorphic to $\mathfrak{H}$, (i.e. $\mathfrak{A}$ with the Jordan product). In $\mathfrak{A}_{j}$, the set of elements $o$-commuting with an element $x$ is just the set of elements commuting with it in the ordinary multiplication, and the double centralizer of $(x)$ for an algebraic element $x$ (over the base field $E$ ) is $(x)$ : the usual proof for matrices over a field goes over for a division ring, since we can quickly reduce it to the case of a cyclic nilpotent matrix $x$ and note that for such an $x$, every matrix commuting with it is a polynomial in $x$ and 1 with coefficients in the division ring, and every matrix commuting with these is a polynomial in $x$ with coefficients in the center $E$ of the division ring. This completes the proof.

THEOREM 2.4. Let $\Im$ be a central simple Jordan algebra and $x$ an

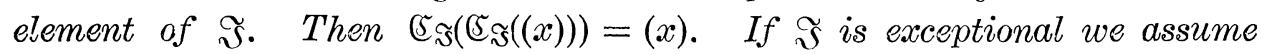
the characteristic is not 2 or 3. 
Proof. Theorem 2.3 covers algebras of types $A, B, C$. Let $\Im$ be of type $D$ : then, by Th. $2.1 \mathrm{~d}$, if $x$ is not in the center of $\Im$ then

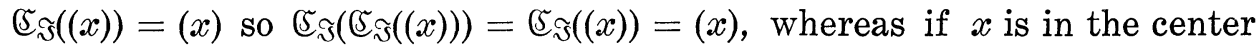
of $\mathfrak{\Im}$, then $\mathfrak{\Im} \mathfrak{s}((x))=\mathfrak{\Im}$, and $\mathfrak{E} \Im(\mathfrak{F} \Im((x)))=$ Center of $\mathfrak{\Im}=(x)$.

It remains to consider the exceptional Jordan algebra $\mathfrak{\Im}$ over an algebraically closed field $F$. If the minimum polynomial of $x$ is of degree three, then by Corollary 2.2, $\sqrt{\Im}((x))=(x)$ so $\sqrt{ } \Im(\sqrt{ } \Im((x)))=(x)$ also. If the minimum polynomial is of degree one, then $(x)=$ Center

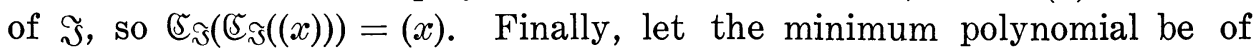
degree two: then there are two cases-minimum polynomial has one root or distinct roots.

Let the minimum polynomial of $x$ be $(x-\gamma 1)^{2}$. Replacing $x$ by $x-\gamma 1$ does not change $(x)$, and so we may assume $x^{2}=0, x \neq 0$. In the proof of Theorem 2.2, we showed that there is a representation of $\Im$ as $H\left(C_{3}\right)$ such that $x$ is represented by the matrix $\alpha\left(e_{11}-e_{22}\right)+e_{12}+e_{21}$, where $\alpha$ is in $F$ and $\alpha^{2}=-1$, and so the elements of $H\left(C_{3}\right) o$-commuting with $x$ are exactly the matrices commuting with the matrix of $x$. We also showed that $e_{3}=(1 / 2) e_{33}$ is in $\mathfrak{E}_{\Im}((x))$. Let $y$ be in $\mathfrak{E}_{\Im}\left(\mathfrak{E}_{\Im}((x))\right)$ : then $y o$-commutes with $e_{3}$ and $y$ is also in $\widetilde{c}_{\Im}((x))$ so that its matrix is as in the proof of Theorem 2.2, and the element $z_{3}$ is zero since $y o$-commutes with $e_{3}$. Thus $y$ also belongs to the simple subalgebra $\Re$ of $H\left(C_{3}\right)$ of elements with coefficients in $F$. Since $y$ o-commutes on $\mathfrak{\Im}$ with every element of $\mathfrak{S}_{\Im}((x))$ and since $\mathfrak{E}_{\Re}((x)) \subseteq \mathfrak{E}_{\Im}((x))$ (each consists of the matrices, in $\Re$ or $\Im$ respectively, commuting with $x$ ), $y o$-commutes on $\mathfrak{\Im}$ with every element of $\widetilde{S}_{\mathscr{S}}((x))$. and in particular $y$-commutes on $\Re$ with every element of $\varsigma_{\Re}((x))$. As $y$ is in $\Re$, we have shown that $y$ is

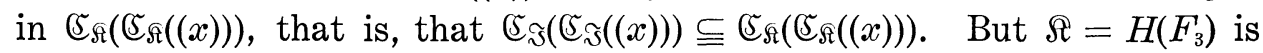
a simple Jordan algebra of degree three and type $B$, and we have already proved for such algebras that $\mathfrak{\complement}_{\mathfrak{N}}\left(\mathfrak{\complement}_{\mathfrak{I}}((x))\right)=(x)$. Thus also $\mathfrak{c}_{\Im}\left(\mathfrak{\complement}_{\Im}((x))\right)=(x)$.

The only case left is that of a minimum polynomial of degree two and distinct roots. Here $x=\alpha e+\beta(1-e)$, $e$ a primitive idempotent and $\alpha \neq \beta$. Clearly $(x)=(e)$, so we may assume $x=e$. $\mathbb{E}_{\mathfrak{s}}((e))=$ $\Im_{e}(0)+\Im_{e}(1)$. Let $y$ be in $\widetilde{\Im}_{\Im}\left(\varsigma_{\Im}((e))\right)$. Then, since $y$ is in $\varsigma_{\Im}((e)), y$ is in $\mathfrak{\Im}_{e}(0)+\mathfrak{\Im}_{e}(1)$ and $y$ o-commutes on $\mathfrak{\Im}$, and therefore on $\mathfrak{\Im}_{e}(0)+\mathfrak{\Im}_{e}(1)$ also, with every element of $\mathfrak{\Im}_{e}(0)+\mathfrak{\Im}_{e}(1)$. Thus $y$ is in the center of this algebra which is the sum of the centers of $\mathfrak{\Im}_{e}(0)$ and $\Im_{e}(1)$, central simple algebras with respective identities $1-e$ and $e$. Thus $y=\gamma(1-e)+\delta e$ belongs to $(e)$, and $\mathfrak{E}_{\mathfrak{s}}\left(\mathfrak{S}_{\Im}((x))\right)=(x)$. This completes the proof.

Section 3. Centralizers of Simple Algebras. In this section we study the centralizer and double centralizer of a simple subalgebra in a central 
simple Jordan algebra. We are also able to study the centralizer theory in certain infinite dimensional Jordan algeberas, namely the algebras of all self-adjoint linear transformations on a self-dual vector space which may be infinite dimensional, such as a Hilbert space. The method is much simpler than that of the last section: we use the enveloping associative algebra and the known centralizer theory of simple associative algebras (see [7] and [15]).

We shall prove analogues of the following theorems on associative algebras ([15]): Let $M, N$ be left and right vector spaces, respectively, over a division ring $D$, dually paired by an inner product $(x, y)$ (i.e. if $(x, z)=0$ for all $z$ in $N$, then $x=0$, and similarly $(u, y)=0$ for all $u$ in $M$ implies $y=0)$. Denote by $\mathfrak{A}=L(M, N)$ the ring of all linear transformations on $M$ having adjoints on $N$, regarded as an algebra over its center. ( $M=N$, that is, $M$ is self-dual, if and only if $\mathfrak{A}$ has an involution ([6]). The involution can be assumed to be the adjoint map.) Let $\mathfrak{B}$ be a simple subalgebra of $\mathfrak{A}$ containing the identity element. Then :

1. $\operatorname{car}(\mathfrak{B})$ is also isomorphic to a ring $L(V, W)$ for a pair of dual spaces $V$ and $W$. If $\mathfrak{A}$ is a simple finite dimensional algebra then so is $\mathfrak{F}_{\mathfrak{A}}(\mathfrak{B})$.

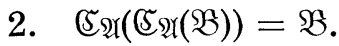

Actually we will also generalize the above associative theorems from rings $L(M, N)$ to the more general primitive rings with minimal ideals, and obtain a corresponding generalization for Jordan algebras. In what follows the term "simple algebra" will be used only for finite-dimensional algebras.

THEOREM 3.1. Let $\Im$ be a special central simple Jordan algebra, $\Re$ a semi-simple subalgebra containing the identity of $\Im$. Then $\mathfrak{C}_{\Im}(\Omega)$ is semi-simple. The same result holds for $\mathfrak{\Im}$ an exceptional algebra provided $\Re$ is separable.

Proof. If $\mathfrak{\Im}$ is of degree one then $\Re=\mathfrak{F}$ and $\mathfrak{s} \Im(\Re)=\mathfrak{F}=\Re$. Next let $\mathfrak{\Im}$ be of degree two. Then $\mathfrak{\Im}=F \cdot 1+V, V a$ vector space with symmetric scalar product. Since $F \cdot 1$ is in $\Re, \Re=F \cdot 1+\Re \cap V$. If $\Re \cap V$ is one-dimensional, then $\mathfrak{c} \Im(\Re)=\Re$; if $\Omega \cap V$ has dimension greater than one, then $\mathfrak{c}_{\Im}(\Re)=F \cdot 1$, and if $\Re \cap V=(0)$ then $\mathfrak{c}_{\mathfrak{s}}(\Re)=\mathfrak{\Im}$ : these statements follow immediately from Theorem 2.1d. Thus $\mathfrak{c}_{\Im}(\Re)$ is semi-simple.

Finally, let $\Im$ have degree three or more, and be special. If $\Im$ is of type $A_{2}$, i.e. isomorphic to $\mathfrak{A}_{j}$ for a central simple associative algebra $\mathfrak{A}$, and $\mathscr{R}$ has enveloping associative algebra $\mathfrak{B}$ in $\mathfrak{A}$, then $\mathfrak{E} \Im(\Re)=\mathfrak{E} \mathfrak{I}(\mathfrak{B})_{j}$. But $\mathfrak{B}$ is a semi-simple associative algebra, therefore $\mathfrak{E}_{\mathfrak{A}(\mathfrak{B})}$ is also semi- 
simple, and so $\left.\mathcal{E}_{2 \mathrm{x}(\mathfrak{B})}\right)_{j}$ is a semi-simple Jordan algebra. The only remaining possibility is that $\mathfrak{S}=H(\mathfrak{U}), \mathfrak{A}$ a simple involutorial algebra. Let $\mathfrak{B}$ be the enveloping algebra of $\Re$. Then $\mathfrak{S} \Im(\Re)=\mathfrak{F} \mathfrak{H}(\mathfrak{B}) \cap \Im=$ set of

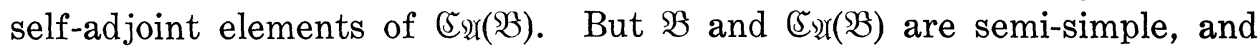
Ear( $(\mathfrak{B})$ is a self-adjoint subalgebra of $\mathfrak{A}$ since $\mathfrak{B}$ is self-adjoint. Therefore the set of self-adjoint elements of $\mathcal{E}_{\text {wr }}(\mathfrak{B})$ is a semi-simple Jordan algebra : ( $\mathfrak{Y r}(\mathfrak{B})$ is a direct sum of simple ideals which are either self-adjoint or interchanged in pairs by the involution, their self-adjoint elements are simple Jordan algebras of types $A_{1}, B, C$ or type $A_{2}$ respectively. This completes the proof for special algebras $\Im$. The exceptional algebra case was proved at the end of Section 1.

As we saw in the above proof, if $\mathfrak{\Im}$ is of type $A_{2}$, i.e. $\mathfrak{\Im}=\mathfrak{A}_{j}$, $\mathfrak{A}$ a central simple associative algebra, and $\Omega$ is a simple subalgebra of $\mathfrak{\Im}$ with enveloping algebra $\mathfrak{B}$, then $\mathfrak{S}_{\mathfrak{s}}(\mathfrak{\Re})=\mathfrak{C}_{\mathfrak{2}(\mathfrak{X}){ }_{j}}$; also, if $\mathfrak{\Im}$ is central simple and $\mathscr{R}$ contains the identity, then $\mathbb{E} \operatorname{sir}\left(\mathbb{E}_{\mathfrak{A}}(\mathfrak{B})\right)=\mathfrak{B}$, so that

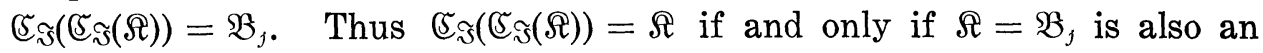
algebra of type $A_{2}$, and the centralizer theory here is identical with the associative theory. The theory for algebras of degree two is not very interesting, since we will always have $\mathfrak{E}_{\mathfrak{s}}(\Re)=\mathfrak{\Im}$, $\Re$, or center of $\mathfrak{\Im}$, as we have seen in the proof of the above theorem.

The remaining type, $\mathfrak{F}=H(\mathfrak{A})$, $\mathfrak{A}$ an involutorial ring of linear transformations, is the only interesting one. We note first of all that the double centralizer of a simple subalgebra $\Omega$ may be actually larger than $\Re$, as is shown by the following examples:

1. Let $\Omega=F \cdot 1+V, V$ even-dimensional, be an algebra of type $D$ and let $\mathfrak{A}$ be the Clifford algebra determined by $V$. We may then assume that $\Re$ is contained in $\mathfrak{A}$. $\mathfrak{A}$ has an involution such that the elements of $\Re$ are self-adjoint; let $\mathfrak{\Im}=H(\mathfrak{U})$, then $\mathfrak{\Im}$ is a simple Jordan algebra of degree greater than two if $V$ has dimension greater than two, and $\mathfrak{\Im}$ properly contains $\Re . \quad \mathfrak{C}_{\Im}(\Re)=F \cdot 1$ since $\Omega$ generates $\mathfrak{A}$, and $\mathfrak{C}_{\Im}(\mathfrak{S} \Im(\Re))=\mathfrak{F}$. We may also embed $V$ in a larger space $W$ with symmetric non-degenerate scalar product, and take $\mathfrak{A}$ to be the Clifford algebra of $W, \mathfrak{I}=H(\mathfrak{U})$. If $\mathfrak{B}$ is the enveloping algebra of $\mathscr{R}$, then $\mathfrak{G}_{\Im}\left(\mathfrak{S}_{\Im}(\Re)\right)$ will contain $H(\mathfrak{B})$, so will be larger than $\Re$. To exclude this possibility we will at least have to assume that if $\mathfrak{B}$ is the enveloping algebra of $\Re$, then $H(\mathfrak{B})=\Re$, that is, that $\Re$ is not of type $D$.

2. Let $\mathfrak{F}=H\left(Q_{n}\right)$, the algebra of $n \times n$ hermitian matrices with quaternion coefficients, $n \geqq 3$, and $\Re$ the subalgebra of $n \times n$ symmetric matrices, $H\left(F_{n}\right)$. It is easy to see that $\mathfrak{c}_{\Im}(\Re)=$ center of $\Im=F \cdot 1$, and $\mathfrak{c} \mathfrak{s}(\mathfrak{S} \mathfrak{s}(\Re))=\mathfrak{\Im}$. Here $\mathfrak{\Im}$ is the set of self-adjoint linear transformations on an $n$-dimensional space and $\Re$ generates an irreducible algebra of linear transformations on the same space, i.e. $\Re$ is almost all of $\Im$. 
To rule out such a case we will have to assume the dimension of the vector space on which $\Im$ operates is not too small relative to $\Re$.

3. Let $\mathfrak{\Im}=H\left(C_{n}\right)$, ordinary $n \times n$ complex-hermitian matrices for a suitably large $n$-an algebra over the field of real numbers, and $\Omega$ the subalgebra of matrices with $r$ equal $s \times s$ symmetric (i.e. with real coefficients) matrix blocks on the main diagonal and zeros elsewhere $(r s=n)$. Then $\Omega$ is isomorphic to $H\left(R_{s}\right)$, and if $r, s \geqq 2$, $\left(\varsigma_{\Im}(\Re)\right.$ is isomorphic to $H\left(C_{r}\right)$, and $\mathbb{E}_{\Im}\left(\mathbb{C}_{\Im}(\Re)\right)$ to $H\left(C_{s}\right)$ which properly contains $\Re$. To exclude this, we will assume that the center of the enveloping associative algebra of $K$ contains the center of the enveloping associative algebra of $\Im$.

We start with a discussion of centralizers of simple subalgebras of a primitive ring with minimal ideals and identity element. Let $M, N$ be dual left and right spaces over the division ring $D$ with center $F$. Then $F \cdot 1$ is the center of the ring $L(M, N)$. Let $F(M, N)$ be the socle of $L(M, N)$, i.e. the linear transformations whose range is finite-dimensional. We will consider primitive rings $\mathfrak{R}$ containing $F \cdot 1$ and such that $L(M, N) \supseteqq \Re \supset F(M, N)$.

Lemma 3.1. Let $\mathfrak{A}$ be a ring of endomorphisms of a module $M$, and $\mathfrak{S}$ a right ideal in $\mathfrak{A}$ such that $M \mathfrak{S}=M$. If $e$ is an endomorphism of $M$ commuting with every element of $\mathfrak{S}$, then $e$ commutes with every element of $\mathfrak{U}$.

Proof. Let $s, a$ belong to $\mathfrak{S}, \mathfrak{A}$ respectively. Then $s(e a-a e)=$ $e s a-s a e$ since $e s=s e$, but since $s a$ is in $\mathfrak{S}, e(s a)=(s a) e$, so $s(e a-a e)$ $=0$ for all $s$ in $\subseteq$. Since $M \subseteq=M, M(e a-a e)=M \subseteq(e a-a e)=0$. Thus $e a=a e$.

THEOREM 3.2. Let $\mathfrak{Y}=L(M, N) \supseteqq \Re \supseteqq F(M, N)+F \cdot 1$, and let $\mathfrak{B}$ be a simple finite-dimensional subalgebra, over $F$, of $\Re$ which contains $F \cdot 1$. Then $\mathbb{C}=\mathfrak{C}_{\mathfrak{R}}(\mathfrak{B})$ is also a primitive ring with minimal ideals and identity element, and $\mathfrak{E}_{\mathfrak{R}}\left(\mathfrak{C}_{\mathfrak{R}}(\mathfrak{B})\right)=\mathfrak{B}$.

Proof. The above theorem is a generalization of a result of Rosenberg, [15], but follows immediately from his result and the above lemma.

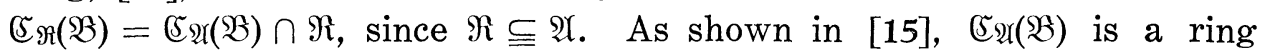
of the form $L(V, W)$ for dual spaces $V$ and $W$, and the socle $\subseteq$ of $\mathfrak{E} \mathfrak{r}(\mathfrak{B})$ is contained in $F(M, N)$. Since $F(M, N) \subset \Re, \quad \mathfrak{S} \subset \mathfrak{R}$, and so $\mathfrak{S} \subset \mathfrak{V}_{\mathfrak{R}}(\mathfrak{B}) \subset \mathfrak{V}_{\mathfrak{l}}(\mathfrak{B})=L(V, W)$. Thus $\mathfrak{V}_{\mathfrak{R}}(\mathfrak{B})$ is a primitive ring with minimal ideals and identity, since $\mathfrak{B}$ and $\mathfrak{R}$ contain the identity of $\mathfrak{A}$. This proves the first statement.

It is known that $M \subseteq=M([15])$. Since $\subseteq$ is a two-sided ideal in

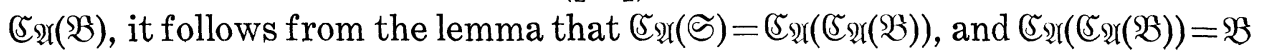




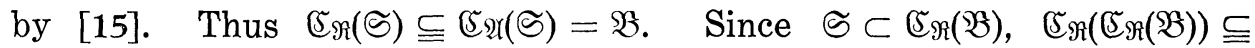
$\mathfrak{E}_{\mathfrak{R}}(\mathfrak{S}) \subseteq \mathfrak{B}$, but $\mathfrak{\Xi}_{\mathfrak{R}}\left(\mathfrak{\Im}_{\mathfrak{R}}(\mathfrak{B})\right) \supseteqq \mathfrak{B}$ always, so $\mathfrak{\Xi}_{\mathfrak{R}}\left(\mathfrak{F}_{\mathfrak{R}}(\mathfrak{B})\right)=\mathfrak{B}$, which we had to prove.

The method of the above proof can be used to extend the Galois theory of rings $L(M, N)$, contained in [16], to primitive rings with minimal ideals.

We state now the main result of this section:

Theorem 3.3. Let $M$ be a self-dual space (see $\S 2$ for the definition) over a division ring $D, E$ the center of $D$, and $F$ the subfield of self-adjoint elements of $E$. Let $\mathfrak{A}=L(M, N), \mathfrak{\Im}=H(\mathfrak{H})$, the Jordan algebra (over $F \cdot 1)$ of self-adjoint linear transformations, and $\Re$ a simple subalgebra of $\mathfrak{\Im}$ of degree greater than two containing $F \cdot 1$ and finite dimensional over it. Assume that $\mathfrak{B}$, the enveloping associative algebra of $\mathfrak{R}$ in $\mathfrak{A}$, contains $E \cdot 1$. Then:

1. If $\Re$ is of type $A_{2}$, thus is isomorphic to $L(V, W)_{j}$ for a pair of dual spaces $V$ and $W$, then $\mathfrak{S} \Im(\Re)$ is isomorphic to $L(P, Q)_{j}$ for a pair of dual spaces $P, Q$.

2. If $\Omega$ is of type $A_{1}, B$ or $C$, i.e. is isomorphic to $H(L(V, V))$ for a self-dual space $V$, then $\mathfrak{\Im}_{\Im}(\Re)$ isomorphic to $H(L(P, P)), P$ self-dual.

3. $\sqrt{\Im}(\mathfrak{\Im} \Im(\Re))=\Re$, provided the dimension of $M$ is greater than twice the dimension over $D$ of any minimal right ideal of $\mathfrak{B} \cdot D$, the ring of endomorphisms of $M$ generated by $\mathfrak{B}$ and the scalar multiplications by elements of: $D$.

Proof. Let $\Omega$ be of type $A_{2}$. Then its enveloping associative algebra $\mathfrak{B}$ is a homomorphic image of the "universal" enveloping algebra $\mathfrak{U}$ of $\mathfrak{R}$, and $\mathfrak{U}=\mathfrak{U}_{1} \oplus \mathfrak{U}_{2}$ where $\mathfrak{H}_{2}$ is anti-isomorphic to $\mathfrak{U}_{1}$ and $\mathfrak{U}_{1}$ and $\mathfrak{U}_{2}$ are simple algebras. Thus either $\mathfrak{B}$ is isomorphic to $\mathfrak{U}_{1}$ or else $\mathfrak{B}$ is isomorphic to $\mathfrak{U}$. $\mathfrak{B}$ is a self-adjoint subalgebra of $\mathfrak{U}$ since $\Re$ consists of selfadjoint elements. Moreover the dimensions over $F$ of $\mathfrak{U}_{1}$ and $\Re$ are the same, so if $\mathfrak{B}$ is isomorphic to $\mathfrak{U}_{1}$ then $\mathfrak{B}=\Re$. Since $\Re$ has degree at least three, $\mathfrak{B}$ is not commutative and so cannot consist only of selfadjoint elements. Thus $\mathfrak{B}=\mathfrak{B}_{1} \oplus \mathfrak{B}_{2}$, where $\mathfrak{B}_{i}$ is isomorphic to $\mathfrak{U}_{i}$. Since $\mathfrak{B}$ is self-adjoint, either $\mathfrak{B}_{1}$ and $\mathfrak{B}_{2}$ are also self-adjoint, or else $\mathfrak{B}_{2}=\mathfrak{B}_{1}^{\prime}$, the image of $\mathfrak{B}_{1}$ under the involution in $\mathfrak{A}$. But if the $\mathfrak{B}_{i}$ are self-adjoint we again get a contradiction: for each $k$ in $\Omega$, write $k=$ $k_{1}+k_{2}, k_{i}$ in $\mathfrak{B}_{i}$ and self-adjoint. Then the map $k$ into $k_{1}$ is a homomorphism of Jordan algebras and so is either zero or an isomorphism since $\Omega$ is simple. If it is an isomorphism it is onto $\mathfrak{B}_{1}$ since $\mathfrak{B}_{1}$ and $\Omega$ have the same dimension over $F$. But then $\mathfrak{B}_{1}$ consists of self-adjoint elements and this is impossible, as before. If the map $k$ into $k_{1}$ is zero then the map $k$ into $k_{2}$ must be onto, which is equally impossible. Hence $\mathfrak{B}=$ $\mathfrak{B}_{1} \oplus \mathfrak{B}_{1}^{\prime}$. 
Let $e_{i}$ be the identity of $\mathfrak{B}_{i}, i=1,2$. Then $e_{2}=e_{1}^{\prime}$ and $e_{1}+e_{2}=1$, $e_{1} e_{2}=0$. Let $M_{i}=M e_{i}$ : then $M=M_{1} \oplus M_{2}$ and the $M_{i}$ are each totally isotropic, for $\left(M e_{i}, M e_{i}\right)=\left(M e_{i} e_{i}^{\prime}, M e_{i}\right)=0$ since $e_{i} e_{i}^{\prime}=0$. As $M$ is selfdual, $M_{1}$ and $M_{2}$ are dually paired by the scalar product in $M$.

Let $\mathfrak{S}=\mathbb{C} \mathfrak{Y}(\mathfrak{B})$. Since $\mathfrak{B}$ contains $e_{1}$ and $e_{2}$, clearly

$\mathfrak{S}=\mathbb{E} \cap e_{1} \mathfrak{A} e_{1} \oplus \mathfrak{S} \cap e_{2} \mathfrak{A} e_{2}=\mathbb{S}_{1} \oplus \mathfrak{S}_{2}$

$=$ centralizer of $\mathfrak{B}_{1}$ in $e_{1} \mathfrak{2} e_{1} \oplus$ centralizer of $\mathfrak{B}_{2}$ in $e_{2} \mathfrak{2} e_{2}$.

Since $\mathfrak{B}_{2}=\mathfrak{B}_{1}^{\prime}, \mathfrak{夭}_{2}=\mathfrak{V}_{1}^{\prime}$. Also, by $\S 3.20$ of [16], $e_{1} \mathfrak{A} e_{1}$ is isomorphic to $L\left(M e_{1}, M e_{1}^{\prime}\right)=L\left(M_{1}, M_{2}\right)$ and in the same way $e_{2} \mathfrak{2}\left(e_{2}\right.$ is isomorphic to $L\left(M_{2}, M_{1}\right) . \quad \mathfrak{夭}_{1}$, being the centralizer in $L\left(M_{1}, M_{2}\right)$ of the simple subalgebra $\mathfrak{B}_{1}$ which contains the center $E \cdot 1$, is a ring $L\left(N_{1}, N_{2}\right)$, by [15], and

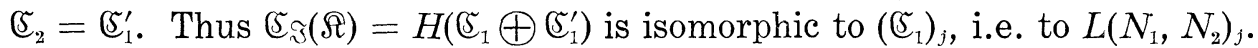
This proves statement 1 .

Let $\Omega$ be of type $A_{2}$ as above, and let $M$ satisfy the dimensionality condition of 3 . Since $E$ is the center of $D$ and $\mathfrak{B}$ contains $E \cdot 1, \mathfrak{B} \cdot D$ is isomorphic to $\mathfrak{B} \bigotimes_{E} D$ and so $\mathfrak{B} \cdot D=\mathfrak{B}_{1} \cdot D \oplus \mathfrak{B}_{2} \cdot D=\mathfrak{B}_{1} \bigotimes_{E} D \oplus$ $\mathfrak{B}_{2} \bigotimes_{E} D$. As the $\mathfrak{B}_{i}$ are simple and contain $E e_{i}$ in their centers, $\mathfrak{B}_{i} \cdot D$ is a simple ring with minimum condition operating on $M_{i}$ and therefore $M_{i}$ is completely reducible and homogeneous as $\mathfrak{B}_{i} \cdot D$ (right) module. By the dimensionality assumption, $M_{i}$ is a direct sum of at least two irreducible submodules. Let $M$ be finite dimensional, then $\mathfrak{E}_{i}$ is the ring of all endomorphisms of $M$ commuting with $\mathfrak{B} \cdot D$ and so is a matrix ring $G_{n}$ over a division ring $G$ with $n \geqq 2$, i.e. $\mathfrak{c}_{1}=L\left(N_{1}, N_{2}\right)$ and $N_{1}$ is a vector space of dimension greater than one. If $M$ is infinite dimensional, so is $N_{1}$. From this it follows that the enveloping ring in $\mathfrak{A}$ of $\mathfrak{F} \cap \mathfrak{\Im}$ contains the socles of $\mathfrak{F}_{1}$ and $\mathfrak{F}_{2}$ : for $\mathfrak{F}_{1}$ is not commutative and so contains elements $a$ and $b$ with $a b \neq b a$. Then $a+a^{\prime}, b+b^{\prime}$ and $b a+a^{\prime} b^{\prime}$ all belong to $\mathfrak{C} \cap \mathfrak{\Im}$, so $\left(a+a^{\prime}\right)\left(b+b^{\prime}\right)-\left(b a+a^{\prime} b^{\prime}\right)=a b-b a$ is in the enveloping ring of $\left(\mathfrak{\Im} \cap \Im\right.$. Also $(a b-b a)\left(g+g^{\prime}\right)=(a b-b a) g$ and $\left(g+g^{\prime}\right)(a b-b a)=g(a b-b a)$ are in this enveloping ring for all $g$ in 5 (since $\mathbb{C} \mathbb{C}^{\prime}=(0)=\mathbb{E}^{\prime} \mathbb{C}$ ), and so this ring contains a non-zero two-sided ideal in $\mathfrak{S}_{1}$. Since every two-sided ideal contains the socle, the enveloping ring does also. If $V, W$ are dual spaces and $\Re$ is a subalgebra of $L(V, W)$ which is the centralizer of a simple finite dimensional subalgebra, then the socle $F$ of $\Re$ satisfies $V F=V$ (see [15]). Therefore if $F_{1}$ is the socle of $\mathfrak{F}_{1}, M_{1} F_{1}=M_{1}$. By Lemma 3.1, the centralizer of $F_{1}$ in

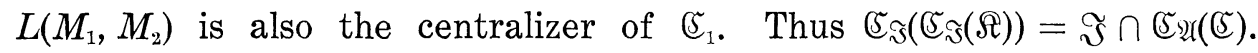
But $\mathfrak{E} \mathfrak{r}(\mathfrak{E})$ is just $\mathfrak{F}_{\mathfrak{r}}\left(\mathfrak{S}_{1} \oplus \mathfrak{S}_{2}\right)$ and the latter is $\mathfrak{B}_{1} \oplus \mathfrak{B}_{2}$ since $\mathfrak{B}_{1}$ is the centralizer of $\mathfrak{S}_{1}$ in $e_{1} \mathfrak{A} e_{1}=L\left(M_{1}, M_{2}\right)$ and similarly for $\mathfrak{B}_{2}$. Therefore $\mathfrak{S}_{\mathfrak{S}}(\mathfrak{C} \Im(\Re))=\mathfrak{B} \cap \mathfrak{\Im}$ and it is known that $\mathfrak{B} \cap \mathfrak{\Im}=H\left(\mathfrak{B}_{1} \oplus \mathfrak{B}_{1}^{\prime}\right)=\Re$ since $\Re$ is of type $A_{2}$.

Next let $\Re$ be of type $A_{1}, B$, or $C$. Then $\mathfrak{B}$ is simple and $\Re=H(\mathfrak{B})$. $\mathfrak{S}_{\Im}(\mathfrak{R})=\mathfrak{G}_{\mathfrak{A}}(\mathfrak{B}) \cap \mathfrak{\Im}$. But $\mathfrak{F}_{\mathfrak{A}}(\mathfrak{B})$ is self-adjoint and so is of the form 
$L(P, P)$ for a self-dual space $P$, since $\mathfrak{B}$ is simple and contains the center $E \cdot 1$ of $\mathfrak{A}$. This proves statement 2.

Let now $M$ satisfy the dimension condition of $3 . \mathfrak{B} \cdot D$ is a simple ring with minimum condition since $\mathfrak{B}$ is simple and contains $E \cdot 1$, and so is homogeneous completely reducible on $M$, and $M$ is a direct sum of at least three irreducible submodules, i.e. $P$ has dimension at least three. Write $\sqrt{5}$ for $(\mathfrak{y a r}(\mathfrak{B})$ and $F$ for the socle of $\mathfrak{E}$. Then $F$ is locally canonically matrix of degree $\geqq 3,[12]$, and so is generated by its selfadjoint elements-i.e. the enveloping ring of $(\mathfrak{s}(\Omega)=H(\mathfrak{S})$ contains $F$.

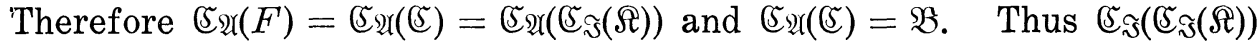
$=\mathfrak{F} \mathfrak{x}(\mathfrak{S}) \cap \mathfrak{F}=\mathfrak{B} \cap \mathfrak{F}$, and finally $\mathfrak{B} \cap \mathfrak{F}=\Re$ since $\mathfrak{\Im}$ is of type $A_{1}, B$ or $C$. Thus $\mathfrak{S}_{\mathfrak{s}}(\mathfrak{S} \Im(\Re))=\Re$. This concludes the proof.

\section{REFERENCES}

1. A. A. Albert, A structure theory for Jordan algebras, Ann. of Math. 48 (1947), 446-467.

2. - A theory of power-associative commutative algebras, Trans. Amer. Math. Soc. 69 (1950), 503-527.

3. C. Chevalley, The algebraic theory of spinors, New York, 1954.

4. J. Dieudonné, Sur la reduction canonique des couples de matrices, Bull. Soc. Math. France, 74 (1946), 130-146.

5. H. Freudenthal, Oktaven, Ausnahmengruppen, und Oktavengeometrie, Utrecht, 1951.

6. N. Jacobson, Lectures in abstract algebra, Vol. 2, New York 1953.

7. — Structure of rings, Amer. Math. Soc., 1956.

8. - Operator commutativity in Jordan algebras, Proc. Amer. Math. Soc., 3 (1952), 973-976.

9. Structure of alternative and Jordan bimodules, Osaka Math. Journal, 6 (1954), 1-71.

10. - On the structure of Jordan algebras, Proc. Nat. Acad. Sci. U.S.A., 42 (1956), $140-147$.

11. N. Jacobson and C. E. Rickart, Jordan homomorphisms of rings, Trans. Amer. Math. Soc., 69 (1950), 479-502.

12. - Homomorphisms of Jordan rings of self-adjoint elements, Trans. Amer. Math. Soc., 72 (1952), 310-322.

13. A. I. Malcev, Foundations of linear algebra, Moscow-Leningrad, 1947.

14. N. H. McCoy, On quasi-commutative matrices, Trans. Amer. Math. Soc., 35 (1934), 332.

15. A. Rosenberg, Finite dimensional simple subalgebras of the ring of all continuous linear transformations, Math. Z. 61 (1954), 150-159.

16. A. Rosenberg and D. Zelinsky, Galois theory of continuous transformation rings, Trans. Amer. Math. Soc., 79 (1955), 429-452.

17. P. Jordan, J. von Neumann, and E. Wigner, On an algebraic generalization of the quantum mechanical formalism, Ann. of Math., 35 (1934), 29-64.

YALE UNIVERSITY 


\section{PACIFIC JOURNAL OF MATHEMATICS}

\section{EDITORS}

\section{David Gilbarg}

Stanford University

Stanford, California

\section{R. A. Beaumont}

University of Washington

Seattle 5, Washington

\author{
A. L. Whiteman
}

University of Southern California Los Angeles 7, California

E. G. Straus

University of California

Los Angeles 24, California

\section{ASSOCIATE EDITORS}

\author{
E. F. BECKENBACH \\ C. E. BURGESS \\ M. HALL \\ E. HEWITT
}
A. HORN
V. GANAPATHY IYER
R. D. JAMES
M. S. KNEBELMAN
L. NACHBIN
I. NIVEN
T. G. OSTROM
H. L. ROYDEN

M. M. SCHIFFER

G. SZEKERES

F. WOLF

K. YOSIDA

\section{SUPPORTING INSTITUTIONS}

\author{
UNIVERSITY OF BRITISH COLUMBIA \\ CALIFORNIA INSTITUTE OF TECHNOLOGY \\ UNIVERSITY OF CALIFORNIA \\ MONTANA STATE UNIVERSITY \\ UNIVERSITY OF NEVADA \\ OREGON STATE COLLEGE \\ UNIVERSITY OF OREGON \\ OSAKA UNIVERSITY \\ UNIVERSITY OF SOUTHERN CALIFORNIA
}

\author{
STANFORD UNIVERSITY \\ UNIVERSITY OF TOKYO \\ UNIVERSITY OF UTAH \\ WASHINGTON STATE COLLEGE \\ UNIVERSITY OF WASHINGTON \\ * * * * \\ AMERICAN MATHEMATICAL SOCIETY \\ CALIFORNIA RESEARCH CORPORATION \\ HUGHES AIRCRAFT COMPANY \\ THE RAMO-WOOLDRIDGE CORPORATION
}

Mathematical papers intended for publication in the Pacific Journal of Mathematics should be typewritten (double spaced), and the author should keep a complete copy. Manuscripts may be sent to any one of the four editors. All other communications to the editors should be addressed to the managing editor, E. G. Straus at the University of California, Los Angeles 24, California.

50 reprints per author of each article are furnished free of charge; additional copies may be obtained at cost in multiples of 50 .

The Pacific Journal of Mathematics is published quarterly, in March, June, September, and December. The price per volume (4 numbers) is $\$ 12.00$; single issues, $\$ 3.50$. Back numbers are available. Special price to individual faculty members of supporting institutions and to individual members of the American Mathematical Society: $\$ 4.00$ per volume; single issues, $\$ 1.25$.

Subscriptions, orders for back numbers, and changes of address should be sent to Pacific Journal of Mathematics, 2120 Oxford Street, Berkeley 4, California.

Printed at Kokusai Bunken Insatsusha (International Academic Printing Co., Ltd.), No. 6, 2-chome, Fujimi-cho, Chiyoda-ku, Tokyo, Japan.

PUBLISHED BY PACIFIC JOURNAL OF MATHEMATICS, A NON-PROFIT CORPORATION

The Supporting Institutions listed above contribute to the cost of publication of this Journal, but they are not owners or publishers and have no responsibility for its content or policies. 


\section{Pacific Journal of Mathematics}

\section{Vol. 8, No. $4 \quad$ June, 1958}

Richard Arens, The maximal ideals of certain functions algebras ........ 641

Glen Earl Baxter, An operator identity ........................... 649

Robert James Blattner, Automorphic group representations ........... 665

Steve Jerome Bryant, Isomorphism order for Abelian groups ............ 679

Charles W. Curtis, Modules whose annihilators are direct summands...... 685

Wilbur Eugene Deskins, On the radical of a group algebra ............ 693

Jacob Feldman, Equivalence and perpendicularity of Gaussian

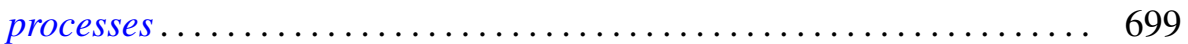

Marion K. Fort, Jr. and G. A. Hedlund, Minimal coverings of pairs by triples....................................... 709

I. S. Gál, On the theory of $(m, n)$-compact topological spaces ......... 721

David Gale and Oliver Gross, A note on polynomial and separable games........................................ 735

Frank Harary, On the number of bi-colored graphs ............... 743

Bruno Harris, Centralizers in Jordan algebras ................... 757

Martin Jurchescu, Modulus of a boundary component ............... 791

Hewitt Kenyon and A. P. Morse, Runs . . . . . . . . . . . . . . . . . . . . . . 811

Burnett C. Meyer and H. D. Sprinkle, Two nonseparable complete metric

spaces defined on $[0,1] \ldots \ldots \ldots \ldots \ldots \ldots \ldots \ldots \ldots \ldots \ldots . \ldots . \ldots . \ldots . \ldots 25$

M. S. Robertson, Cesàro partial sums of harmonic series expansions...... 829

John L. Selfridge and Ernst Gabor Straus, On the determination of numbers by their sums of a fixed order ........................ 847

Annette Sinclair, A general solution for a class of approximation

problems .................................

George Szekeres and Amnon Jakimovski, $(C, \infty)$ and $(H, \infty)$ methods of summation...................................... 867

Hale Trotter, Approximation of semi-groups of operators. ............. 887

L. E. Ward, A fixed point theorem for multi-valued functions ........... 921

Roy Edwin Wild, On the number of lattice points in $x^{t}+y^{t}=n^{t / 2} \ldots \ldots .929$ 